\title{
Smoothened is a master regulator of adult liver repair
}

\author{
Gregory A. Michelotti, ${ }^{1}$ Guanhua Xie, ${ }^{1}$ Marzena Swiderska, ${ }^{1}$ Steve S. Choi, ${ }^{1}$ \\ Gamze Karaca, ${ }^{1}$ Leandi Krüger, ${ }^{1}$ Richard Premont, ${ }^{1}$ Liu Yang, ${ }^{1}$ Wing-Kin Syn, ${ }^{1,2}$ \\ Daniel Metzger, ${ }^{3}$ and Anna Mae Diehl'
}

\author{
1Division of Gastroenterology, Department of Medicine, Duke University Medical Center, Durham, North Carolina, USA. \\ 2The Institute of Hepatology, Regeneration and Repair, The Foundation for Liver Research, London, United Kingdom. \\ ${ }^{3}$ Department of Functional Genomics and Cancer, Institut de Génétique et de Biologie Moléculaire et Cellulaire, INSERM, Paris, France.
}

\begin{abstract}
When regenerative processes cannot keep pace with cell death, functional epithelia are replaced by scar. Scarring is characterized by both excessive accumulation of fibrous matrix and persistent outgrowth of cell types that accumulate transiently during successful wound healing, including myofibroblasts (MFs) and progenitors. This suggests that signaling that normally directs these cells to repair injured epithelia is deregulated. To evaluate this possibility, we examined liver repair during different types of liver injury after Smoothened (SMO), an obligate intermediate in the Hedgehog ( $\mathrm{Hh})$ signaling pathway, was conditionally deleted in cells expressing the MF-associated gene, $\alpha S M A$. Surprisingly, blocking canonical Hh signaling in MFs not only inhibited liver fibrosis but also prevented accumulation of liver progenitors. Hh-sensitive, hepatic stellate cells (HSCs) were identified as the source of both MFs and progenitors by lineage-tracing studies in 3 other strains of mice, coupled with analysis of highly pure HSC preparations using flow cytometry, immunofluorescence confocal microscopy, RT-PCR, and in situ hybridization. The results identify SMO as a master regulator of hepatic epithelial regeneration based on its ability to promote mesenchymal-to-epithelial transitions in a subpopulation of HSC-derived MFs with features of multipotent progenitors.
\end{abstract}

\section{Introduction}

Cirrhosis, a lethal end point of all chronic liver diseases, is increasing at an alarming rate due to recent epidemics of chronic viral hepatitis and obesity-related liver disease (1). Cirrhosis develops when regenerative processes fail to keep pace with liver cell death, leading to progressive replacement of functional epithelia with scar $(2,3)$. Liver fibrosis is the hallmark of cirrhosis, and fibrosis (i.e., scarring) is thought to drive the changes in liver function and blood flow that cause liver-related morbidity and mortality $(3,4)$. Because medical interventions targeted at reversing excessive accumulation of fibrous matrix have been unsuccessful, liver transplantation is the only curative treatment available. Transplantation is precluded in the vast majority of cirrhotic individuals, however, due to the relative dearth of donor organs. This gap between demand and supply is predicted to widen (1), and deaths from cirrhosis will increase unless treatments to improve regeneration of chronically injured livers are developed. Success will require better understanding of the mechanisms that control reconstruction of functional liver epithelia during chronic injury.

Scarring is characterized by both excessive accumulation of collagen matrix and persistent outgrowth of cell types that accumulate transiently during successful wound healing, including MFs and progenitors $(1,5)$. Both cell types mediate key facets of liver

Authorship note: Gregory A. Michelotti and Guanhua Xie contributed equally to this work.

Conflict of interest: The authors have declared that no conflict of interest exists.

Note regarding evaluation of this manuscript: Manuscripts authored by scientists associated with Duke University, The University of North Carolina at Chapel Hill, Duke-NUS, and the Sanford-Burnham Medical Research Institute are handled not by members of the editorial board but rather by the science editors, who consult with selected external editors and reviewers.

Citation for this article: J Clin Invest. 2013;123(6):2380-2394. doi:10.1172/JCI66904 repair. In addition to producing and resorbing matrix, MFs are an important source of trophic factors for liver epithelial progenitors $(5,6)$. The latter cells, in turn, not only differentiate to replace dead liver epithelial cells, but also generate soluble factors that control MF populations $(7,8)$. Because MFs and liver epithelial progenitors accumulate together in areas of scarring, autocrine/paracrine signaling that normally directs these cells to repair injured liver epithelia may be deregulated in cirrhosis. Identifying the precise signaling pathway(s) and particular cell type(s) that drive deregulated repair has been challenging due to the plethora of injury-related factors and resultant complexity of wound-healing responses.

Hedgehog (Hh), a pleiotropic, morphogenic-signaling pathway that orchestrates tissue growth during fetal development, is active in adult livers during injury of various etiologies $(9,10)$. Hh signaling is tightly regulated in adulthood. Healthy livers barely express Hh ligands, and neither mature hepatocytes nor cholangiocytes exhibit active Hh signaling (11-13). Both types of mature liver epithelial cells begin to produce Hh ligands, however, when subjected to lethal stresses or certain injury-associated cytokines $(7,14)$. These ligands diffuse away from the wounded, ligand-producing epithelial cells, entering the bile canaliculi and liver sinusoids to activate viable Hh-responsive cells that line these structures. The latter include resident pericytes (also known as hepatic stellate cells [HSCs]) in the space of Disse and progenitors along the canals of Hering. Hh pathway activation stimulates HSCs to become proliferative, fibrogenic myofibroblasts (MFs). It also promotes the growth and survival of liver progenitor populations. Both HSC-derived MFs (MF-HSCs) and liver progenitors, in turn, also produce Hh ligands, further enriching the injured hepatic microenvironment with these factors (10).

Hh ligands interact with the membrane-spanning receptor, Patched (PTC), on the surface of Hh-responsive cells, thereby silencing PTC inhibition of the signaling competent corecep- 
tor, Smoothened (SMO). Activated SMO promotes stabilization and nuclear localization of glioma (Gli) family transcription factors that regulate the expression of Hh target genes controlling cell viability, growth, and differentiation (9). In addition to Hh ligand-PTC interactions, SMO activity is modulated via multiple pathways, including $G$ protein-coupled receptors and TGF- $\beta$ / TGF- $\beta$-receptor interactions (15-17). Gli factors are also controlled by SMO-independent (i.e., noncanonical) mechanisms that are initiated by other injury-related factors, including the key profibrogenic cytokine, TGF- $\beta$ (16-18).

The relative significance of canonical SMO-dependent $\mathrm{Hh}$ signaling and SMO-independent mechanisms for proper regeneration of damaged adult livers is unknown. However, emerging evidence supports important roles for canonical Hh signaling in adult liver repair. Studies in mice treated systemically with pharmacologic antagonists of SMO show that acute activation of the canonical Hh pathway is necessary to regenerate liver epithelia (19) but also indicate that persistent SMO activation promotes scarring (20). The basis for these differential effects of canonical Hh signaling on liver repair is not known. Further study of this issue is justified because replacing injured liver epithelia without scarring appears to depend upon appropriate regulation of SMO activity.

\section{Results}

Conditional disruption of SMO in $\alpha \mathrm{SMA}^{+}$cells disrupts Hh signaling in MFs. To investigate the role of Hh-responsive MFs in adult liver repair, we used the Cre-LoxP system to disrupt SMO function in cells expressing the MF-associated gene $\alpha$-smooth muscle actin ( $\alpha S M A)$. Because resident HSCs are both Hh-responsive and a major source of MFs during liver injury, the efficacy of Cre-mediated SMO recombination was tested first in primary HSCs that were harvested from SMO-flox mice, cultured to permit their transition into MFs, and then treated with control adenovirus expressing GFP or adenovirus harboring Cre recombinase. Compared with freshly isolated (day 0) HSCs, HSCs that were cultured and then treated with AdGFP demonstrated increased expression of Smo, several Hh-regulated genes (Gli1, Gli2, and Ptc), the pro-epithelial-to-mesenchymal transition (pro-EMT) transcription factor, SNAIL, and MF genes, such as $\alpha S M A$ and collagen $1 \alpha 1$ (Col1a1). In contrast, expression of genes that antagonize EMT $(\mathrm{Bmp} 7$, E-cadherin $[\mathrm{CDH}]$, and desmoplakin $[D s p]$ ) and genes that mark HSC quiescence (Gfap and Pparg) was lower than that in freshly isolated HSCs. Treating culture-activated HSCs with AdCre significantly suppressed Smo and reduced mRNA expression of Hh target genes, Snail, and MF genes, while genes that antagonize EMT were induced, and markers of HSC quiescence were upregulated (Figure 1A). As expected, knocking down SMO in MF-HSCs prevented recombinant Sonic hedgehog ligand from inducing Hh-regulated transcription factors or increasing expression of MF genes (Supplemental Figure 1; supplemental material available online with this article; doi:10.1172/ JCI66904DS1). The aggregate data, therefore, validate the efficacy of this approach for inhibiting SMO and prove that SMO activation is required for MF-HSCs to accomplish canonical Hh signaling and maintain a profibrogenic phenotype in culture.

In addition, the data support the inherent plasticity of HSCs by demonstrating that such cells coexpress epithelial and mesenchymal genes and showing that HSCs modulate the relative expression levels of these genes in response to environmental cues, such as $\mathrm{Hh}$ ligands. Expression of epithelial genes predominates when HSCs are quiescent, but mesenchymal gene expression becomes dominant after Hh pathway activation. Indeed, freshly isolated HSCs (marked by desmin) coexpress E-cadherin, which localizes along the interface between closely adjacent HSCs (Supplemental Figure 2A). Although cadherin junctions between HSCs in healthy livers had been reported previously (21-23), it is not generally accepted that HSCs have epithelial properties. Therefore, we used immunohistochemistry to confirm that E-cadherin colocalized with desmin in uninjured livers of adult mice (Supplemental Figure 2B). The results prove that quiescent HSCs (Q-HSCs) are relatively epithelial in intact tissue and when freshly isolated but transition to become myofibroblastic when Hh signaling is activated during culture. In cultured MF-HSCs, knocking down SMO abrogates Hh signaling, and this apparently permits the cells to revert back toward their more epithelial phenotype. Hence, in vitro, HSCs are transitional cells that are capable of EMT/mesenchymal-to-epithelial transition (EMT/MET). Subsequent studies aimed to determine whether or not similar transitions might occur in vivo.

Double-transgenic (DTG) mice were generated by crossing SMO-flox mice with $\alpha$ SMA-Cre-ER ${ }^{\mathrm{T} 2}$ mice, in which the $\alpha \mathrm{SMA}$ promoter drives expression of tamoxifen-dependent (TMX-dependent) Cre recombinase-estrogen receptor fusion protein (24). TMX drives Cre recombinase into the nucleus to delete the floxed SMO exon, thereby abrogating Hh signaling selectively in $\alpha$ SMAexpressing cells. To examine the effect of Hh signaling in MFs in vivo, DTG mice were subjected to sham surgery or bile duct ligation (BDL) for 14 days to promote hepatic accumulation of MFs derived from portal fibroblasts (PFs), HSCs, and bone marrow fibrocytes $(25,26)$. Between days 4 and 10 , half of the mice in each group were treated with TMX and half were treated with vehicle. PCR analysis of whole liver DNA demonstrated the absence of detectable transgene rearrangement in vehicle-treated mice but substantial loss of the floxed SMO allele and accumulation of the deleted allele in TMX-treated mice (Figure 1B). Whole liver SMO expression fell significantly (Figure 1C and Supplemental Figure 3), showing that DTG mice can be used to determine whether Hh signaling in MFs is important for liver repair in vivo.

Consistent with the fact that healthy livers exhibit little $\mathrm{Hh}$ pathway activity, GLI2 (Figure 1D) and SMO (Supplemental Figure 3) expression was negligible in sham-operated mice and not influenced by TMX. Also as predicted by evidence that BDL activates hepatic Hh signaling, BDL increased Smo (Figure 1C and Supplemental Figure 3) and induced nuclear accumulation of GLI2 in vehicle-treated DTG mice. TMX significantly inhibited this (Figure 1, C and D, and Supplemental Figure 3), leading to decreased mRNA expression of the GLI2 target gene, Gli1, and suppression of various GLI1-regulated genes, including frizzled-related peptide 1 (Frp1) and osteopontin (Opn) (Figure 1E). Thus, conditional disruption of SMO in $\alpha$ SMA-expressing cells inhibits Hh signaling in vivo.

Unexpectedly, reduced nuclear GLI2 was observed in ductular cells and periportal hepatocytic cells in DTG mice that were treated with TMX after BDL (Figure 1D). Immature ductular cells and periportal hepatocytes are derived from bipotent liver epithelial progenitors that reside along periportal canals of Hering (27). Therefore, we evaluated livers of sham-operated and BDL mice for expression of $\alpha$-fetoprotein (AFP), a marker of hepatocytic progenitors. Livers of sham-operated DTG controls demonstrated only rare $\mathrm{AFP}^{+}$cells (data not shown). In contrast, BDL-treated DTG mice that received vehicle after BDL exhibited numerous AFP-expressing cells in and 

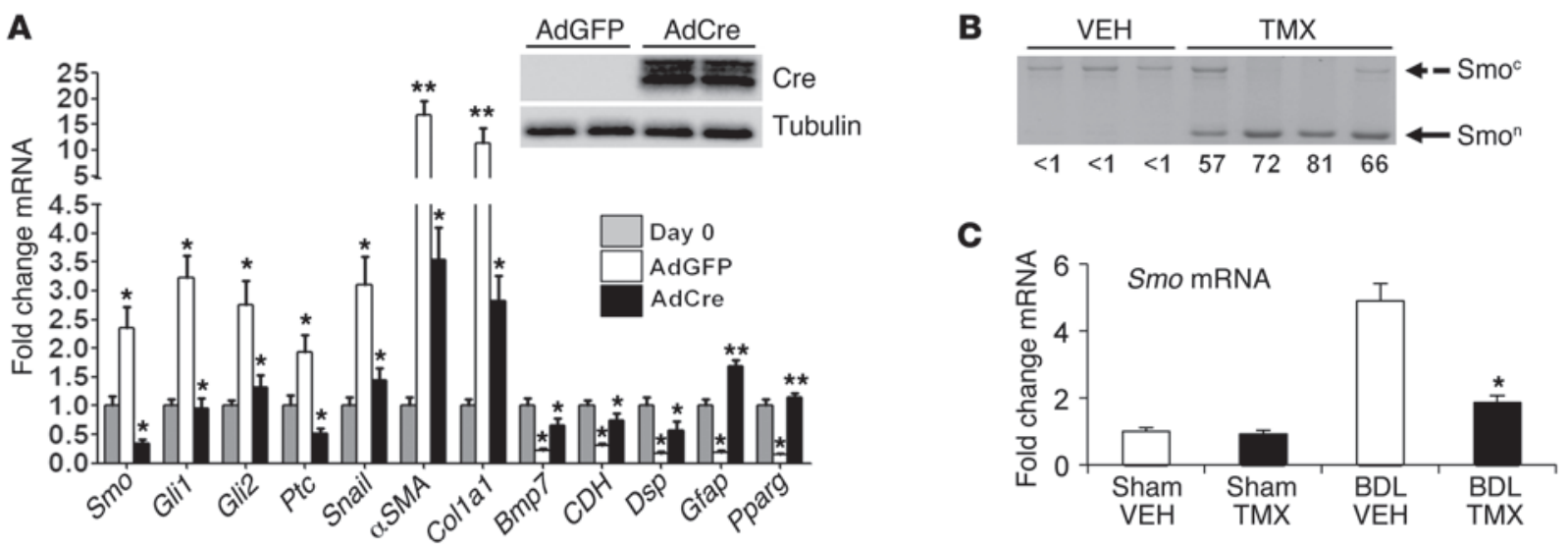
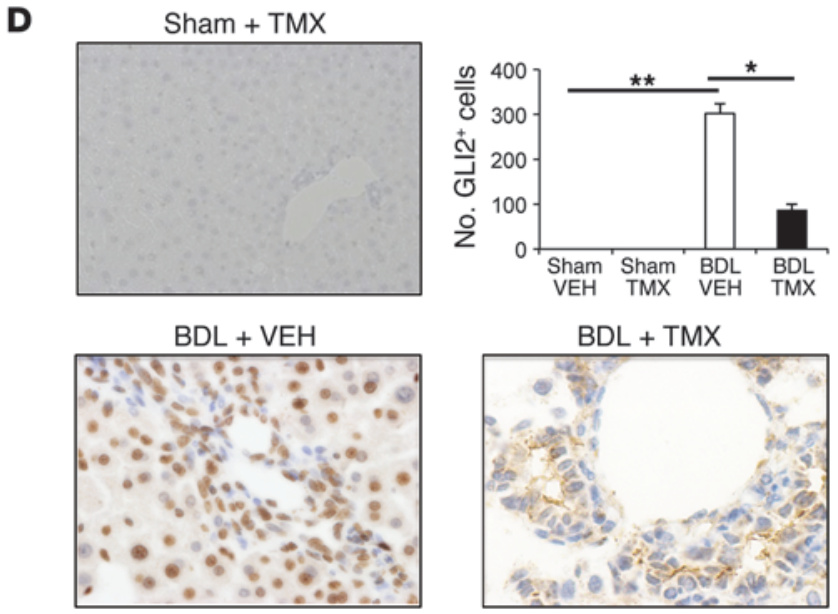

$\mathbf{E}$

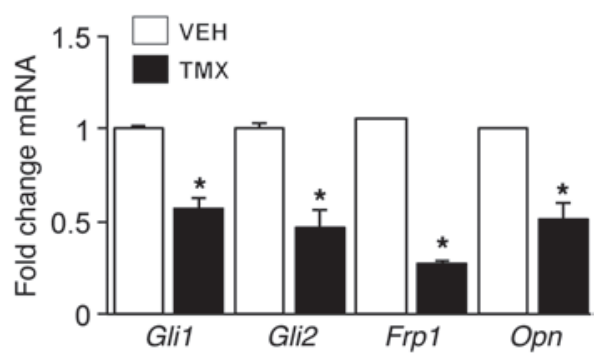

\section{Figure 1}

Conditional disruption of SMO in $\alpha \mathrm{SMA}^{+}$cells inhibits Hh signaling in MFs. (A) HSCs were isolated from Smo-flox mice. AdGFP or AdCre (MOI 25) were added on culture day 4 , and cells were harvested 3 days later. Effects on gene expression were assessed by qRT-PCR. Results are normalized to day 0 HSCs. ${ }^{*} P<0.05$. Inset shows representative Western blot for expression of Cre and tubulin. (B) Oligonucleotides for the floxed SMO allele (refs. 56, 63; Supplemental Table 3) were used to distinguish the unrearranged allele (Smoc; $1.7 \mathrm{~Kb})$ from the Cre-recombined null allele (Smon; $0.5 \mathrm{~Kb})$. Genomic DNA was isolated from livers of DTG mice treated either with vehicle (VEH, $n=3)$ or TMX $(n=4)$ from day 4-10 after BDL, and $\mathrm{Smo}^{n}$ and $\mathrm{Smo}^{\circ}$ amplicons were amplified. Quantification of the amplicons was performed by densitometry to assess the efficiency of Cre-mediated recombination. Results are expressed as $S m o^{n} / S_{m o n}+S_{0} o^{c} \times 100$, shown below each lane. (C) Total RNA was isolated from livers of vehicle- and TMX-treated DTG mice 14 days after sham surgery or BDL. SMO expression was assessed by qRT-PCR, and results are expressed as fold over sham plus vehicle control. ${ }^{\star} P<0.05$. (D) Representative immunohistochemistry for hepatic GLI2. GLI2+ cells in vehicle-treated and TMX-treated DTG mice were quantified in sham and BDL groups and represented as number of positive cells per field ( $n=11$ /group; original magnification, $\times 20$ [Sham]; $\times 63$ [BDL]). ${ }^{*} P<0.05,{ }^{* *} P<0.01$. (E) Total RNA was isolated from livers of vehicle- and TMX-treated DTG mice 14 days after BDL and analyzed by QRT-PCR for expression of Hh-regulated genes. Results are expressed as fold over vehicle-treated group. ${ }^{*} P<0.05$.

around portal tracts (Supplemental Figure 4). Colocalization of GLI2 was noted in many $\mathrm{AFP}^{+}$cells, including AFP-expressing hepatocytic cells that emerged from canals of Hering-like structures. Some AFP-negative hepatocytic cells and ductular cells, as well as many stromal cells, also expressed GLI2. In contrast, DTG mice that received TMX after BDL had reduced numbers of GLI2 ${ }^{+}$cells and failed to accumulate $\mathrm{AFP}^{+}$cells after BDL.

Blocking Hh signaling in MFs inhibits fibrosis and accumulation of MFs and ductular cells in injured livers. BDL provokes a fibrotic response that enlarges portal tracts relative to uninjured liver (Figure 2, Supplemental Figures 5 and 6, and ref. 28). This occurred in vehicle-treated DTG mice but was significantly attenuated in TMXtreated DTG mice (Figure 2A and Supplemental Figure 6). Mor- phometry of Sirius red-stained liver sections (Figure 2B), whole liver collagen mRNA expression (Figure 2C), and hepatic hydroxyproline content (Figure 2D) confirmed reduced liver fibrosis.

The fibrotic reaction to biliary obstruction involves periportal accumulation of various types of MFs and ductular cells (4). Therefore, we compared numbers of cells expressing $\alpha$ SMA, a general marker for MFs (6), or keratin 19 (Krt19), a marker for ductular cells (29), in the 2 groups. Vehicle-treated DTG mice accumulated large numbers of $\alpha$ SMA- and Krt19-positive cells after BDL. Accumulation of both cells types was significantly inhibited in TMX-treated DTG mice. Decreases in aSMA and Krt19 by immunohistochemistry were paralleled by reduced $\alpha S M A$ and Krt19 mRNAs (Figure 3). 
A
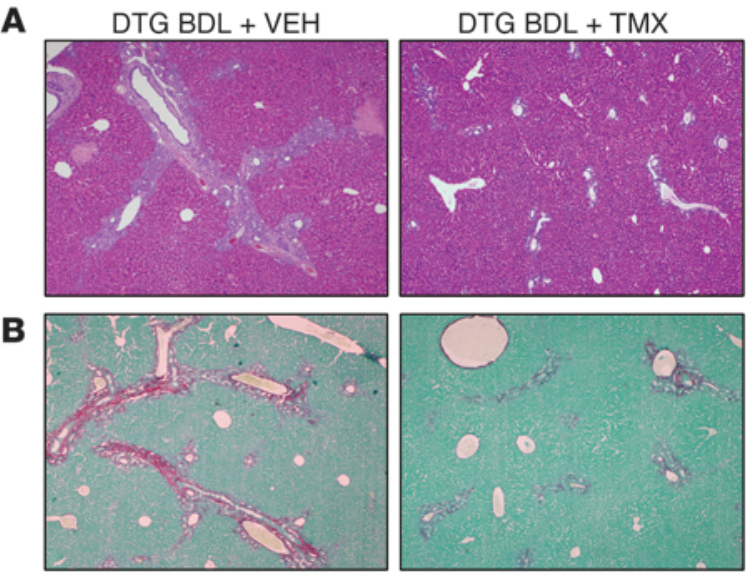

\section{C}

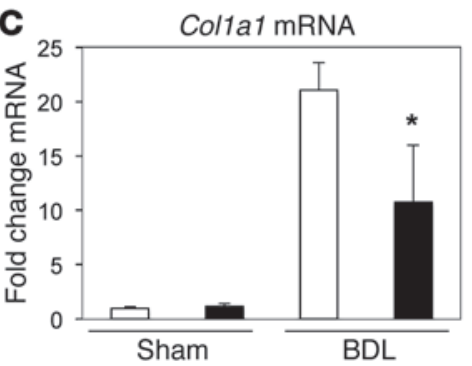

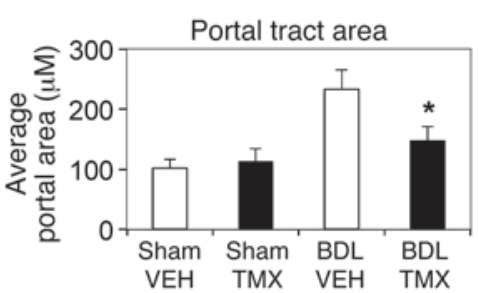

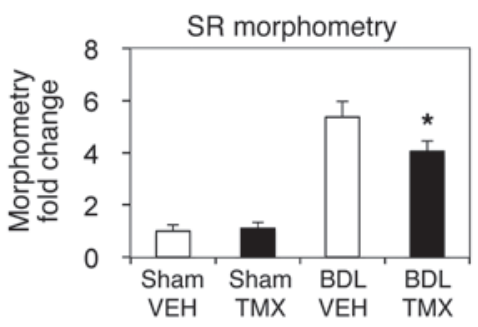

\section{Figure 2}

Blocking $\mathrm{Hh}$ signaling inhibits portal tract expansion and fibrosis in injured livers. (A) H\&E staining of liver sections from representative vehicle- and TMX-treated DTG mice 14 days after sham surgery or BDL (original magnification, $\times 4$ ). Portal tract size was evaluated by morphometric analysis of H\&E-stained sections from all mice ( $n=4$ mice in sham vehicle; $n=4$ mice in sham TMX; $n=11$ mice in BDL vehicle; $n=11$ mice BDL TMX), expressed as mean \pm SEM of portal tract size, and graphed relative to vehicle-treated sham-operated mice. ${ }^{*} P<0.05$ vs. vehicle-treated sham group. (B) Other sections were stained with Sirius red/fast green to demonstrate fibrosis (original magnification, $\times 4$ ). Morphometric data are displayed as mean \pm SEM. ${ }^{*} P<0.05$ vs. vehicle-treated DTG mice after BDL. (C) Total liver RNA was isolated, and Col1a1 mRNA levels were analyzed by qRT-PCR. Results are expressed as fold over vehicle-treated sham-operated DTG group. ${ }^{*} P<0.05$ vs. vehicle-treated BDL group. (D) Quantification of hepatic hydroxyproline in the respective groups. Results are expressed relative to vehicle-treated sham-operated DTG group. ${ }^{*} P<0.05$ vs. vehicle-treated BDL group.

MFs in BDL livers derive from 2 main sources, HSCs and PFs (4, 28). Therefore, liver sections were stained for desmin, a marker of MFHSCs (30), or elastin, a marker of PFs (31). Vehicle-treated mice accumulated large numbers of both desmin ${ }^{+}$and elastin ${ }^{+}$cells after BDL (Figure 3). Accumulation of both cell types was significantly reduced in TMX-treated DTG mice and was paralleled by similar repression of the respective mRNAs (Figure 3). As we observed when Smo was conditionally deleted in cultured MF-HSCs (Figure 1A), deleting $\mathrm{SMO}$ in $\alpha \mathrm{SMA}^{+}$cells after BDL increased expression of various EMT inhibitors and upregulated Q-HSC genes (Supplemental Figure 7) and E-cadherin (Supplemental Figure 2B), suggesting that the fate of MF-HSCs in intact tissue was altered by disrupting Hh signaling.

Blocking $\mathrm{H} h$ signaling in MFs inhibits accumulation of liver epithelial progenitors in injured livers and causes liver atropby. Ductular cells are derived from multipotent progenitors that express the transcription factor SOX9 (32-35). BDL induced significant accumulation of SOX9 ${ }^{+}$cells in vehicle-treated DTG mice but not in TMX-treated DTG mice (Figure 4A and Supplemental Figure 8). As shown earlier (Supplemental Figure 4), TMX blocked accumulation of cells that expressed AFP (Figure 4B), a marker of hepatocyte precursors, and decreased mRNA expression of Sox9, $A f p$, and various stem cell markers, such as CD133 and Nanog (Figure 4C).
The liver/body weight ratios of TMX-treated DTG mice were also somewhat reduced after BDL (Figure 4D). SMO deletion might have promoted relative liver atrophy by exacerbating liver injury after BDL, but BDL-related increases in serum alanine aminotransferase and numbers of TUNEL cells were not exacerbated in the TMXtreated group (Figure 4D). Also, TMX did not increase infarction or congestion in the liver (Figure 2A and Supplemental Figures 5 and 6) or extrahepatic organs (e.g., lung or kidney; Supplemental Figures 9 and 10), as might have been expected if $\alpha \mathrm{SMA}^{+}$vascular smooth muscle cells were altered. Therefore, it is not likely that failure to restore liver mass resulted from altered blood flow or increased liver cell death. Rather, we noted significantly fewer hepatocytes and ductular cells with nuclear staining for Ki67, a marker of proliferation (Figure 4E). Hh pathway activation stimulates expression of cyclin D1 and FOXM1, factors that facilitate cell cycle progression and that are required for liver regeneration after partial hepatectomy (36, 37). Compared with vehicle-treated DTG mice, numbers of cyclin D1-expressing cells and whole liver expression of cyclin D1 (Ccnd1) and Foxm 1 mRNAs (Figure 4, F and G) were significantly reduced in TMX-treated DTG mice after BDL. Therefore, abrogating SMOdependent signaling in $\alpha \mathrm{SMA}^{+}$cells mainly seemed to inhibit regenerative responses to cholestatic liver injury. 


\section{DTG BDL + VEH}

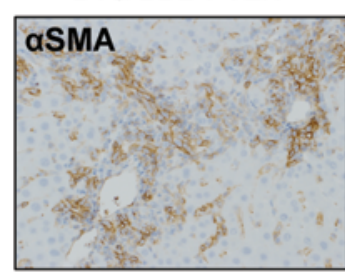

DTG BDL + VEH

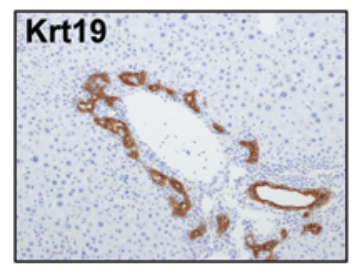

DTG BDL + VEH

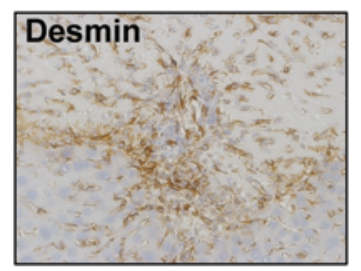

DTG BDL + VEH

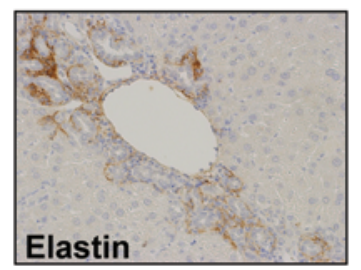

DTG BDL + TMX

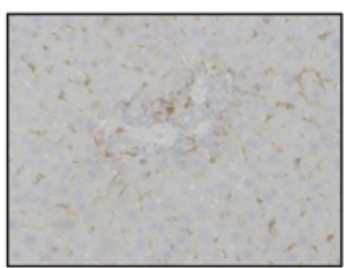

DTG BDL + TMX

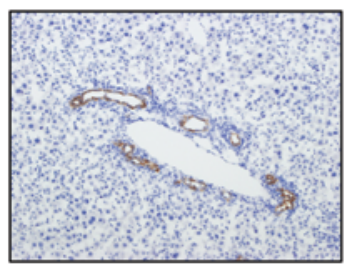

DTG BDL + TMX

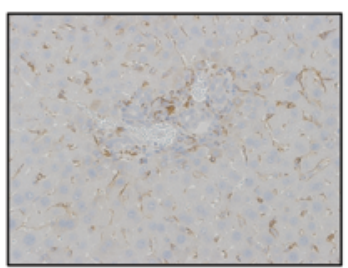

$\mathrm{DTG} B \mathrm{BD}+\mathrm{TMX}$

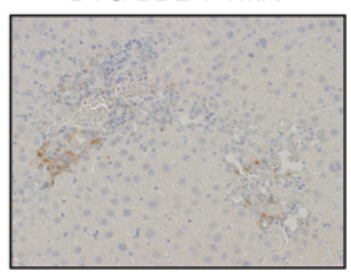

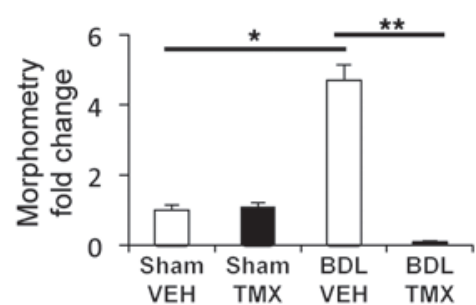
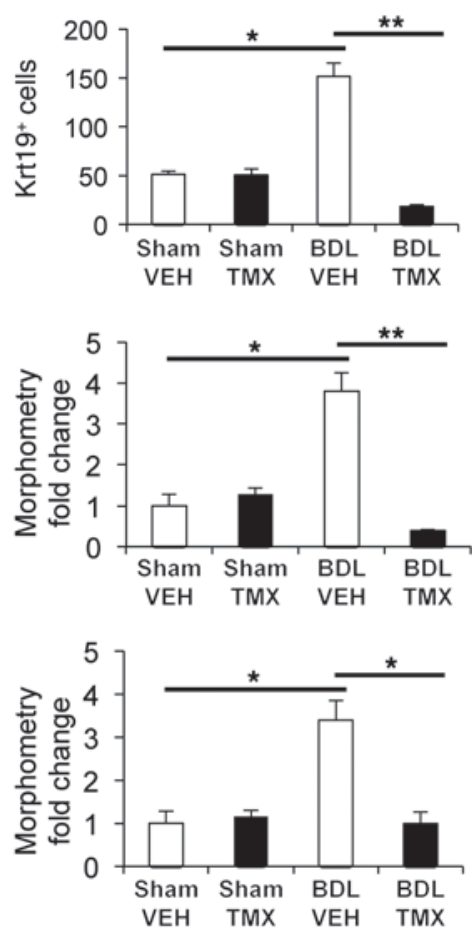
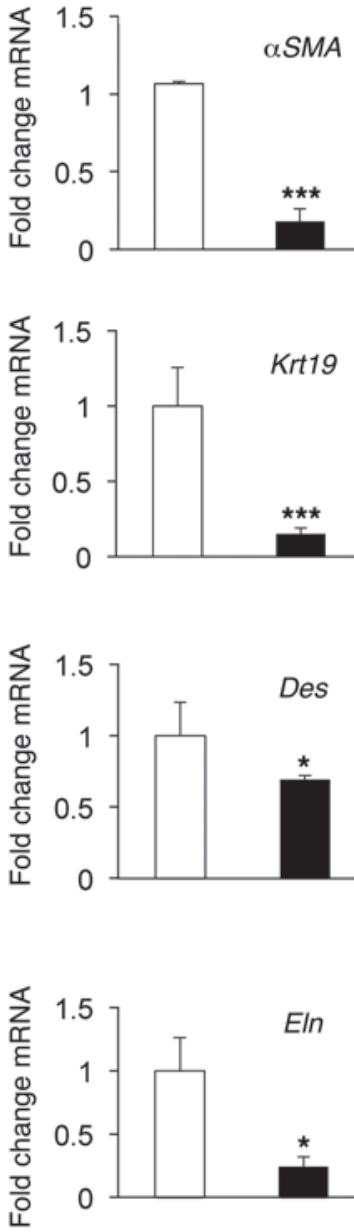

\section{Figure 3}

Blocking Hh signaling in MFs inhibits injury-induced accumulation of MFs and ductular cells. DTG mice were treated with either vehicle or TMX following sham surgery or BDL, as described in Methods. Representative immunohistochemistry, whole liver morphometry, and whole liver mRNA expression for $\alpha \mathrm{SMA}$, Krt19, desmin, and elastin are shown. Results are expressed either as fold over vehicle-treated sham-operated controls (computer-assisted morphometry) or total numbers of positively stained cells per field $(n=11 /$ group; original magnification, $\times 20)$. ${ }^{*}<<0.05$; ${ }^{* *} P<0.001$. qRT-PCR analysis of gene expression in whole liver total RNA isolated from vehicle- (white bars) or TMX-treated (black bars) BDL groups is shown. Results are expressed as fold over vehicle-treated BDL group. ${ }^{*} P<0.05$; ${ }^{* * *} P<0.01$.

To further investigate the significance of Hh-responsive MFs in progenitor-mediated liver repair, we fed DTG mice methionine choline-deficient, ethionine-supplemented (MCDE) diets for 1 week to stimulate hepatic lipid accumulation, injure hepatic parenchymal cells, inhibit hepatocyte replication, and trigger compensatory outgrowth of MFs and immature liver epithelial cells (8). As expected, this provoked hepatic steatosis (Supplemental Figure 11) and triggered significant hepatic accumulation of aSMA-expressing cells (Figure 5A), desmin ${ }^{+}$cells (Figure 5B), and progenitor cells (Figure 5, C and D) in vehicle-treated DTG mice. Although TMX had no effect on any of these markers in DTG mice fed control diets (data not shown) and did not attenuate MCDE diet-induced steatosis in DTG mice (Supplemental Figure 11), it significantly blocked accumulation of cells expressing $\alpha$ SMA, desmin, AFP, and SOX9 and reduced mRNA expression of these markers in MCDE diet-fed DTG mice (Figure 5, A-D). As in BDL mice (Figure 4D), inhibiting MF and progenitor accumulation in
MCDE diet-fed mice reduced liver/body weight ratios (Figure 5E). Also consistent with findings in BDL mice, loss of SMO resulted in decreased expression of the Hh-regulated gene Gli2, as well as various other MF genes (Col1a1, elastin, and vimentin) and progenitor markers (Krt7 and Krt19; Supplemental Figure 12). Therefore, in mouse models of chronic biliary injury (BDL) and chronic hepatocyte injury (MCDE diets), failure to expand liver MF populations correlated with restricted outgrowth of liver epithelial progenitors and caused liver atrophy. This might have resulted from lack of MF-derived trophic factors or depletion of a pool of MFs that function as liver epithelial precursors.

Fate mapping demonstrates that both epithelial and stromal cells are progeny of cells that express HSC markers. To differentiate between these possibilities, we crossed $\alpha$ SMA-Cre-ER ${ }^{\mathrm{T} 2}$ mice with ROSA-Stopflox-YFP mice (38) to generate another DTG line in which TMX could be used to mark $\alpha$ SMA-expressing cells and their progeny with YFP. Immunohistochemistry demonstrated that expression 
A

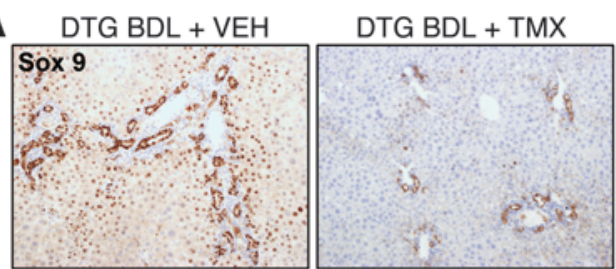

C
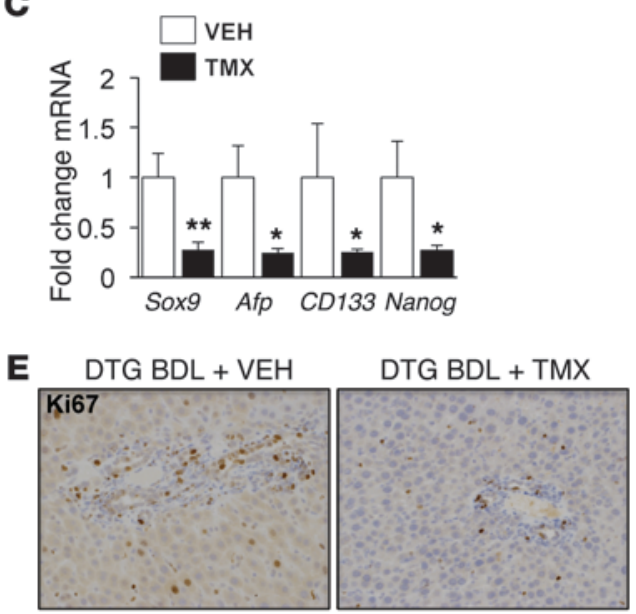

F $\mathrm{DTG} B D L+\mathrm{VEH}$

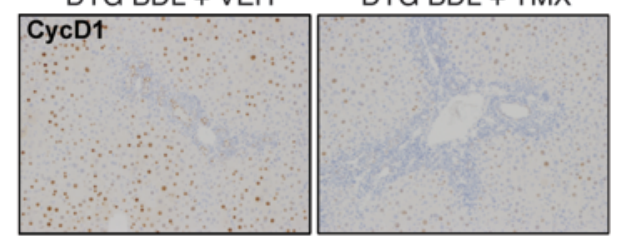

B

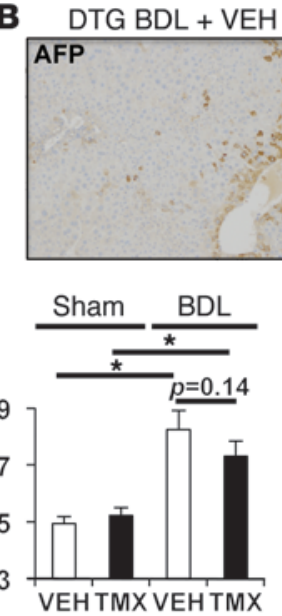

DTG BDL + TMX
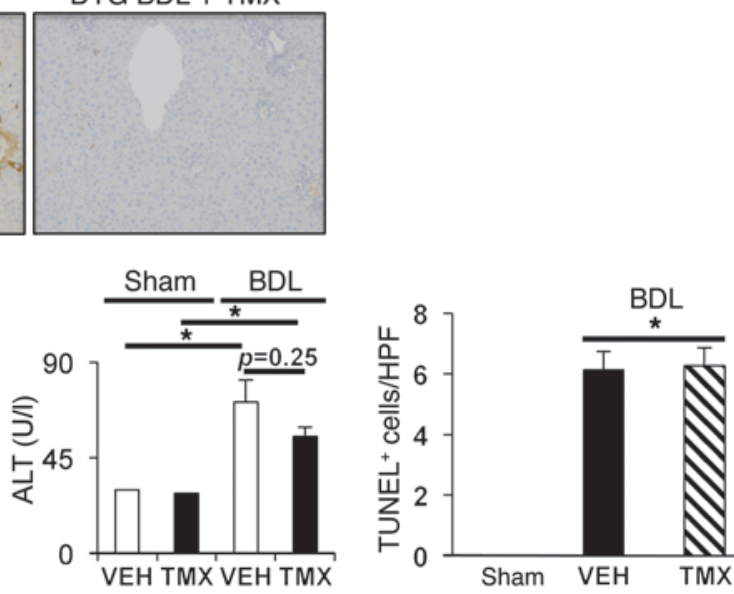

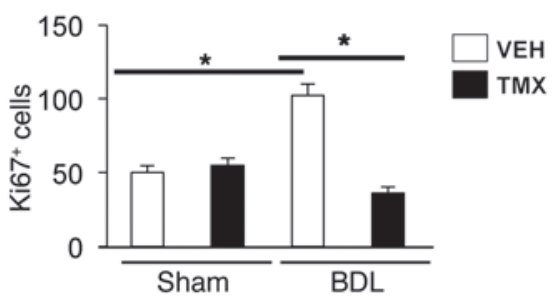

G
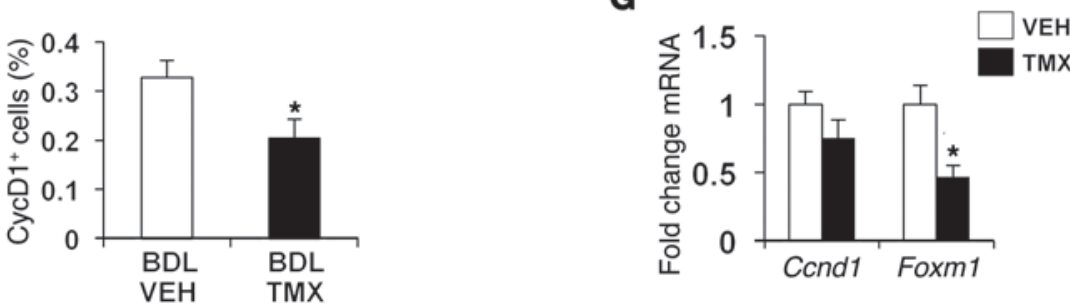

Figure 4

Blocking Hh signaling in MFs inhibits accumulation of liver epithelial progenitors, causes liver atrophy, and blocks liver cell proliferation after BDL injury. (A) SOX9 protein expression in representative immunostained liver sections from vehicle-treated or TMX-treated DTG mice 14 days after BDL (original magnification, $\times 20$ ). (B) Representative liver sections immunostained for AFP (original magnification, $\times 20$ ). (C) qRT-PCR analysis of Sox9, Afp, CD133, and Nanog in whole liver RNA of DTG animals. Results are expressed as mean \pm SEM relative to vehicle-treated BDL group. ${ }^{* *} P<0.01$; ${ }^{*} P<0.05$. (D) Liver/body weight (LW/BW) ratios, serum levels of alanine aminotransferase (ALT), and TUNEL-positive cells in intact liver tissue 14 days after BDL in DTG mice treated with vehicle or TMX. Results are graphed as mean \pm SEM. ${ }^{*} P<0.05$. (E) Ki67 staining in representative vehicle-treated and TMX-treated mice 14 days after BDL. Ki67-positive cells were quantified and graphed as mean \pm SEM relative to the vehicle-treated sham-operated group. ${ }^{*} P<0.05$ vs. vehicle-treated BDL group (original magnification, $\left.\times 20\right)$. (F) Representative cyclin D1 (CycD1) staining is similarly shown, with cyclin D1-positive cells graphed as mean \pm SEM relative the vehicle-treated sham-operated group (original magnification, $\times 20$ ). ${ }^{*} P<0.05$ vs. vehicle-treated BDL group. (G) qRT-PCR analysis of cyclin D1 (Ccnd1) and Foxm1 expression in whole liver mRNA. Results in TMX-treated BDL group are shown relative to results in vehicle-treated BDL group (mean $\pm \mathrm{SEM}) .{ }^{*} P<0.05$.

of $\alpha$ SMA in the new DTG strain mirrored that of WT mice, both following sham surgery and BDL (Supplemental Figure 13). YFP staining was not demonstrated in WT mice or in DTG mice that were treated with vehicle (Figure 6A and Supplemental Figure 13). However, YFP-marked cells were demonstrated when DTG mice were treated with TMX to activate Cre recombinase (Figure 6A and Supplemental Figure 13). Critically, many more $\mathrm{YFP}^{+}$cells accumulated in such mice after BDL than after sham surgery. YFP-marked cells localized within 3 different tissue compartments in injured livers: ductular structures, stroma, and hepatocyte plates (Figure $6 \mathrm{~A}$ and Supplemental Figure 13). To assess whether these findings might have resulted from misregulation of Cre recombinase and/or faulty antibody specificity, hepatocytes were isolated from the vari- ous groups and further analyzed by flow cytometry and direct fluorescence (Figure 6B). Gene rearrangement in hepatocytes was also evaluated by PCR analysis (Figure 6C). Both approaches confirmed the immunohistochemistry data, with all assays showing that at least one-quarter of hepatocytes were derived from $\alpha$ SMA-expressing cells (range 24\%-34\%, Figure 6, B and C, and Supplemental Figure 14), thus supporting the hypothesis that Hh-responsive MF populations include precursors of adult hepatocytes.

aSMA is a general marker of MFs and as such is expressed by MFs derived from bone marrow fibrocytes, PFs, and HSCs (31, 39). To differentiate which of these myofibroblastic cell types might have given rise to liver epithelial cells during liver injury, we generated a third DTG line in which TMX could be used to condi- 


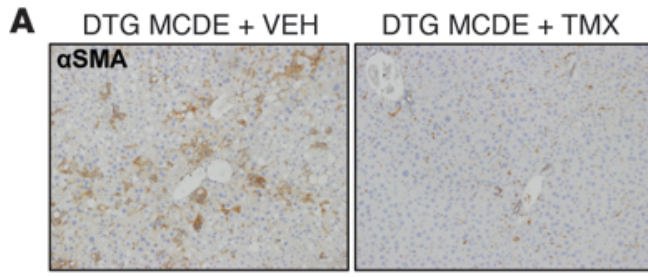

B $D T G M C D E+V E H$

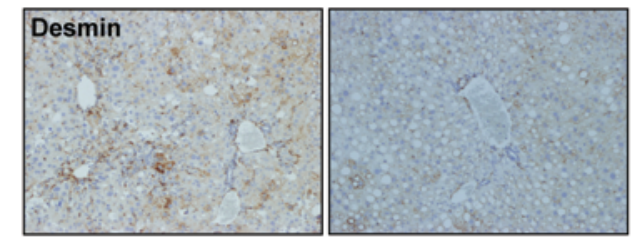

C $\mathrm{DTG} M \mathrm{MCDE}+\mathrm{VEH}$

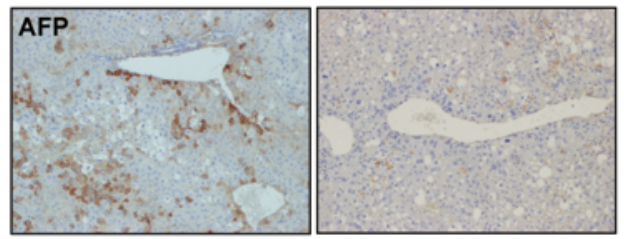

D $\mathrm{DTG} M C D E+\mathrm{VEH}$

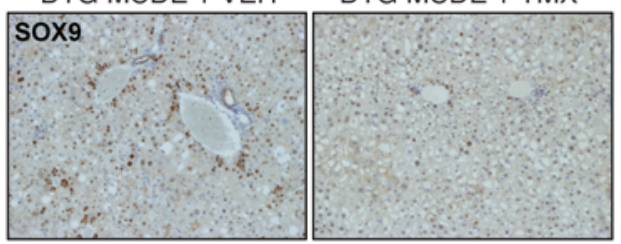

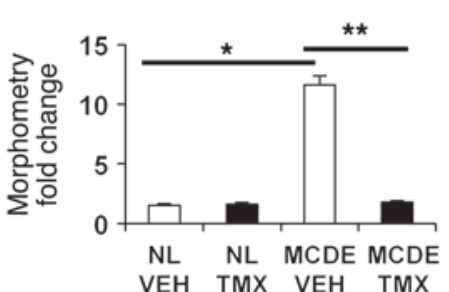
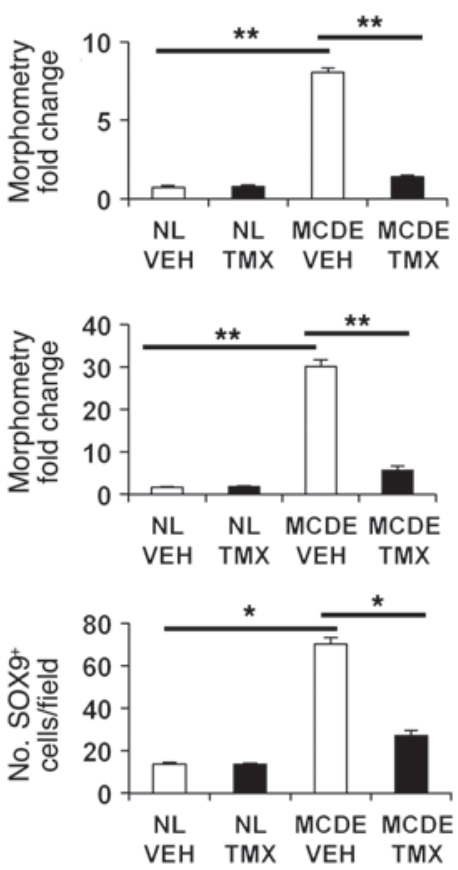
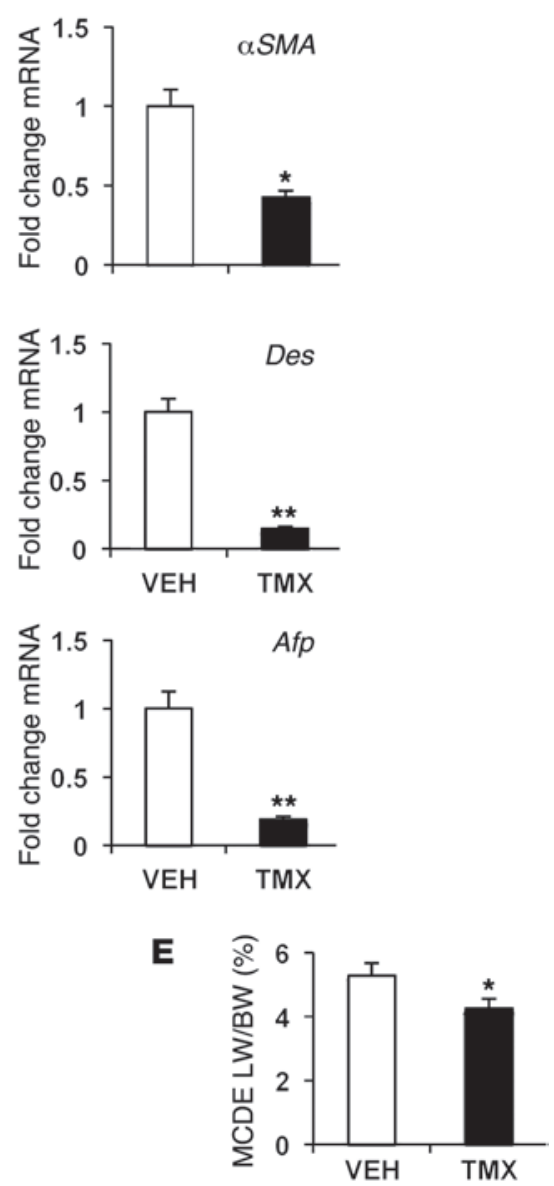

Figure 5

Blocking Hh signaling in MFs inhibits accumulation of liver epithelial progenitors, causes liver atrophy, and blocks liver cell proliferation after MCDE diet-induced injury. (A-D) Representative immunohistochemistry, liver morphometry data, and whole liver mRNA expression for (A) aSMA, (B) desmin, (C) AFP, and (D) SOX9 in DTG mice following MCDE diet-induced injury. Mice were fed MCDE diets for 1 week, with each group receiving injections of either vehicle or TMX on days $0,2,4$, and 6 , and livers were harvested on day 7 . Results are expressed either as fold over vehicle-treated sham-operated controls (computer-assisted morphometry) or total numbers of positively stained cells per field (original magnification, $\times 20)$. NL, normal. ${ }^{*} P<0.05 ;{ }^{\star *} P<0.01$. Results of qRT-PCR analysis of gene expression in the MCDE groups are shown, with results expressed as fold over vehicle-treated MCDE group. ${ }^{*} P<0.05 ;{ }^{* *} P<0.01$. (E) Representative liver/body weight ratios, 7 days after MCDE in DTG mice treated with vehicle or TMX. Results are graphed as mean \pm SEM. ${ }^{*} P<0.05$.

tionally mark GFAP-expressing cells and their progeny with YFP. Because GFAP is expressed by HSCs, but not bone marrow fibrocytes or PFs (31), GFAP-Cre-ERTM/ROSA-Stop-flox-YFP DTG mice will differentiate liver epithelial cells that arise from HSCs (YFP positive) from those that might have been derived from PFs or fibrocytes (YFP negative). When the GFAP-Cre-ERTM/ROSAStop-flox-YFP DTG mice were subjected to BDL to provoke expansion of both HSC-derived and non-MF-HSCs (Figure 3), we observed striking accumulation of YFP-marked hepatocytes and ductular cells (Figure 6D and Supplemental Figure 15), proving that GFAP-expressing cells were precursors of at least some liver epithelial cells. These findings mirrored the results of the lineage-tracing studies in $\alpha$ SMA-Cre-ER ${ }^{\mathrm{T} 2} /$ ROSA-Stop-flox-YFP mice (Figure 6A and Supplemental Figure 13) and extended the findings in cultured HSCs (Figure 1 and Supplemental Figure 2), together supporting a role for HSCs as precursors of liver epithelial cells in BDL-injured livers, because HSCs are the only type of adult liver cell that is known to express both GFAP and $\alpha$ SMA.
FACS and fate-mapping evidence that HSCs and MF-HSCs are reprogrammable. The concept that HSCs are capable of differentiating into hepatocytes and ductular cells is contrary to current dogma but not entirely novel. Some other groups have reported that HSCs can express various progenitor markers, including SOX9, in vitro $(40,41)$. We showed that $\mathrm{Hh}$ signaling differentially modulates HSC expression of epithelial and mesenchymal markers, and our prior lineage-tracing studies of constitutive GFAP-Cre/ROSAStop-flox-YFP and GFAP-Cre/ROSA-Stop-flox-LacZ mice during MCDE-induced liver injury demonstrated that hepatocytes and cholangiocytes derive from cells that expressed GFAP (8). However, other workers have been unable to demonstrate the occurrence of epithelial-mesenchymal transitions during adult liver injury $(42,43)$. Therefore, to further investigate the possibility that some MF-HSCs in injured livers might become liver epithelial cells, we used FACS analysis (Figure 7, A-E, and Supplemental Figure 16, A and B), RT-PCR (Figure 8A and Supplemental Figure 17), and immunofluorescence plus confocal microscopy (Figure 
A
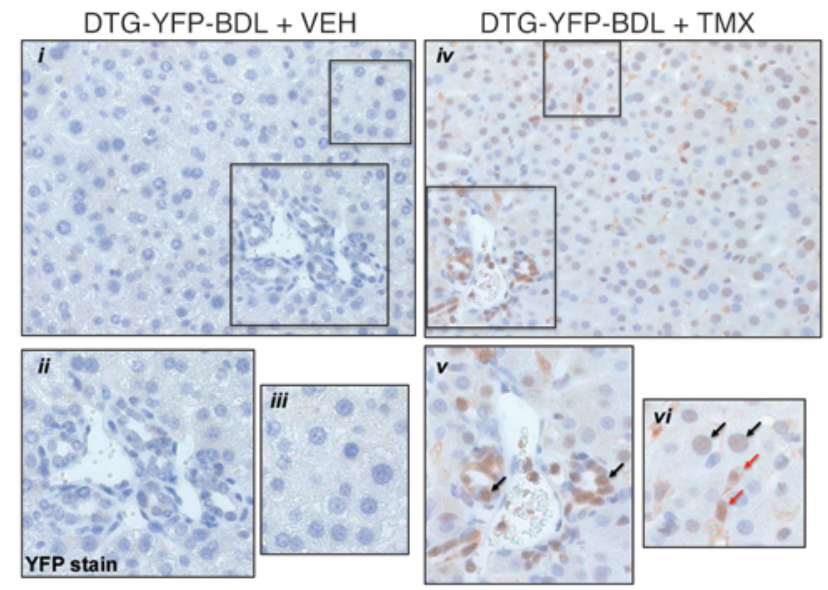

C

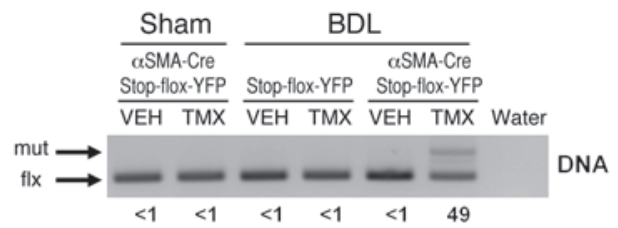

Rosa-Stop-Flox EYFP locus

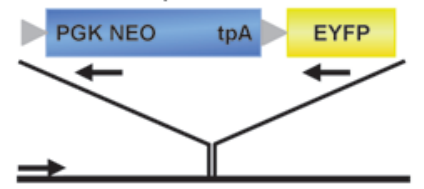

ROSA26 locus

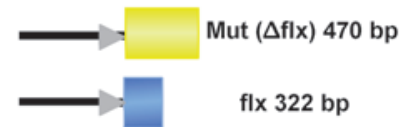

D
B

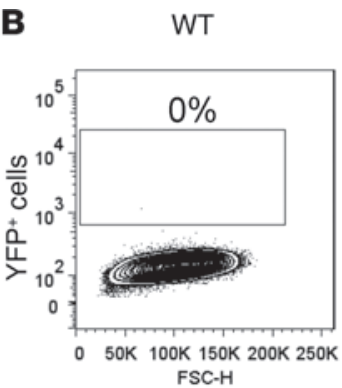

$\alpha$ SMA-Cre/Stop-flox-YFP Sham + TMX
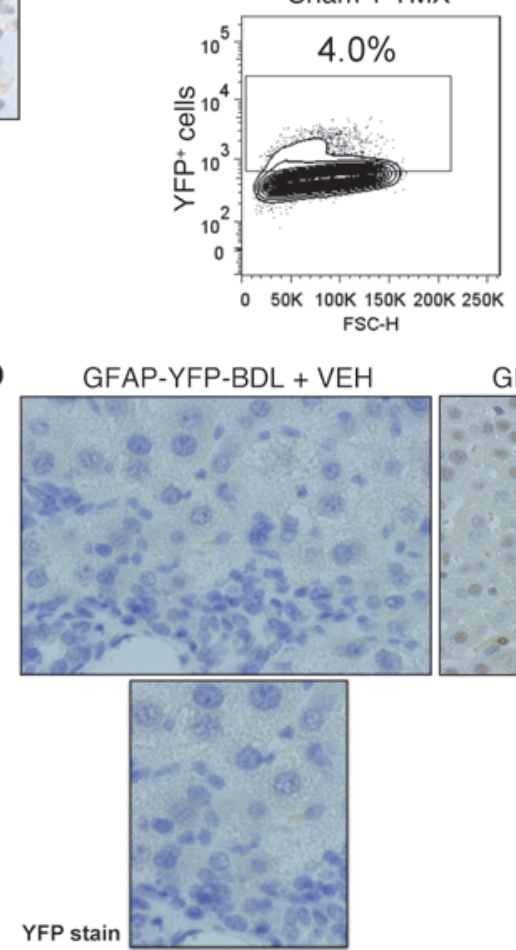
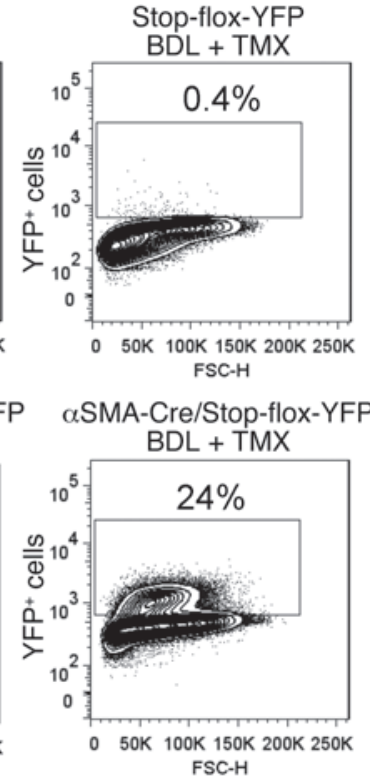

aSMA-Cre/Stop-flox-YFP

GFAP-YFP-BDL + TMX

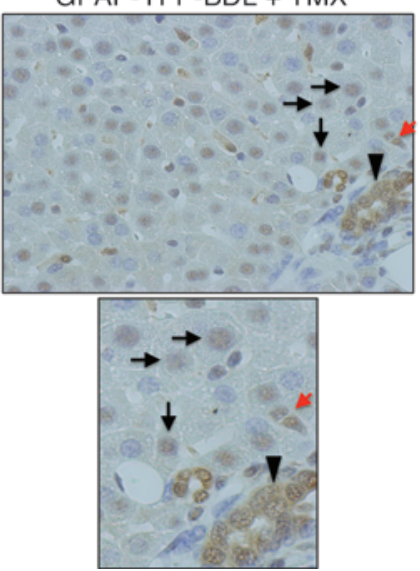

\section{Figure 6}

Epithelial and stromal cells are progeny of cells that express markers of HSCs. (A) $\alpha$ SMA-Cre-ER ${ }^{\top 2} \times$ ROSA-Stop-flox-YFP mice were subjected to sham surgery or 14-day BDL and treated with either vehicle or TMX. Immunohistochemistry for YFP was performed, and representative images are shown (original magnification, $\times 40[i-i v]$ ). Boxed regions show increased magnification of representative YFP staining of ductular cells in periportal areas ( $\mathrm{v}$; arrows) and hepatocytic and stromal cells in midzonal areas (vi; black and red arrows, respectively; original magnification, $\times 100$ [ $\mathrm{v}$ and vi]). Vehicle-treated controls are also shown (ii and iii, respectively). (B) Wild-type, ROSA-Stop-flox-YFP (no Cre), and $\alpha$ SMA-Cre-ER ${ }^{\text {T2/ROSA- }}$ Stop-flox-YFP (DTG-YFP) mice were subjected to sham surgery or BDL and treated with vehicle or TMX. Primary hepatocytes were isolated 14 days after BDL and analyzed for direct YFP fluorescence by flow cytometry. The percentages of YFP-positive cells relative to WT control are indicated. (C) Hepatocytes were isolated from ROSA-Stop-flox-YFP and DTG-YFP mice; DNA was analyzed by PCR to examine rearrangement of the ROSA26 locus (gray triangles depict LoxP sites). A common forward primer was used with 2 downstream primers specific for the indicated regions (PGK Neo, EYFP; Supplemental Table 3). Cre-mediated recombination was quantified as described in Figure 1 and shown below each lane. (D) Lineage tracing was also performed in GFAP-Cre-ERTM x ROSA-Stop-flox-YFP mice subjected to 14-day BDL injury and treated with vehicle or TMX. Immunohistochemistry for hepatic YFP was performed, and representative images are shown of YFP+ cells in ductular (arrowhead), stromal (red arrow), and hepatocytic (black arrows) regions (original magnification, $\times 40$ [top]; ×100 [bottom]).

8B) to profile primary HSCs that were isolated from healthy adult WT mice and analyzed immediately (day 0 ) or after culture for 7 days. HSC isolates were stained with BODIPY to demonstrate lipid globules and analyzed by violet laser to identify vitamin $\mathrm{A}^{+}$cells according to the protocol of Kisseleva and Brenner (44). $98 \%$ of our freshly isolated HSCs were positive for BODIPY and vitamin A (Supplemental Figure 16C). 97\%-98\% of the cells also expressed the HSC marker, desmin, further assuring that both fresh isolates and cultured cells were highly pure (Figure 7A). As expected, we found that certain proteins were differentially expressed in the day 0 HSCs and the culture-activated (day 7) HSCs. Most notably, $98 \%$ of day 7 HSCs expressed the MF-HSC marker, $\alpha$ SMA, whereas fewer than $7 \%$ of day 0 HSCs expressed this protein (Figure 7A). FACS analysis for PTC and GLI2 also confirmed that Hh signaling was much more active in MF-HSCs than in freshly isolated HSCs (Figure 7B). Surprisingly, SOX9 was consistently demonstrated 
research article
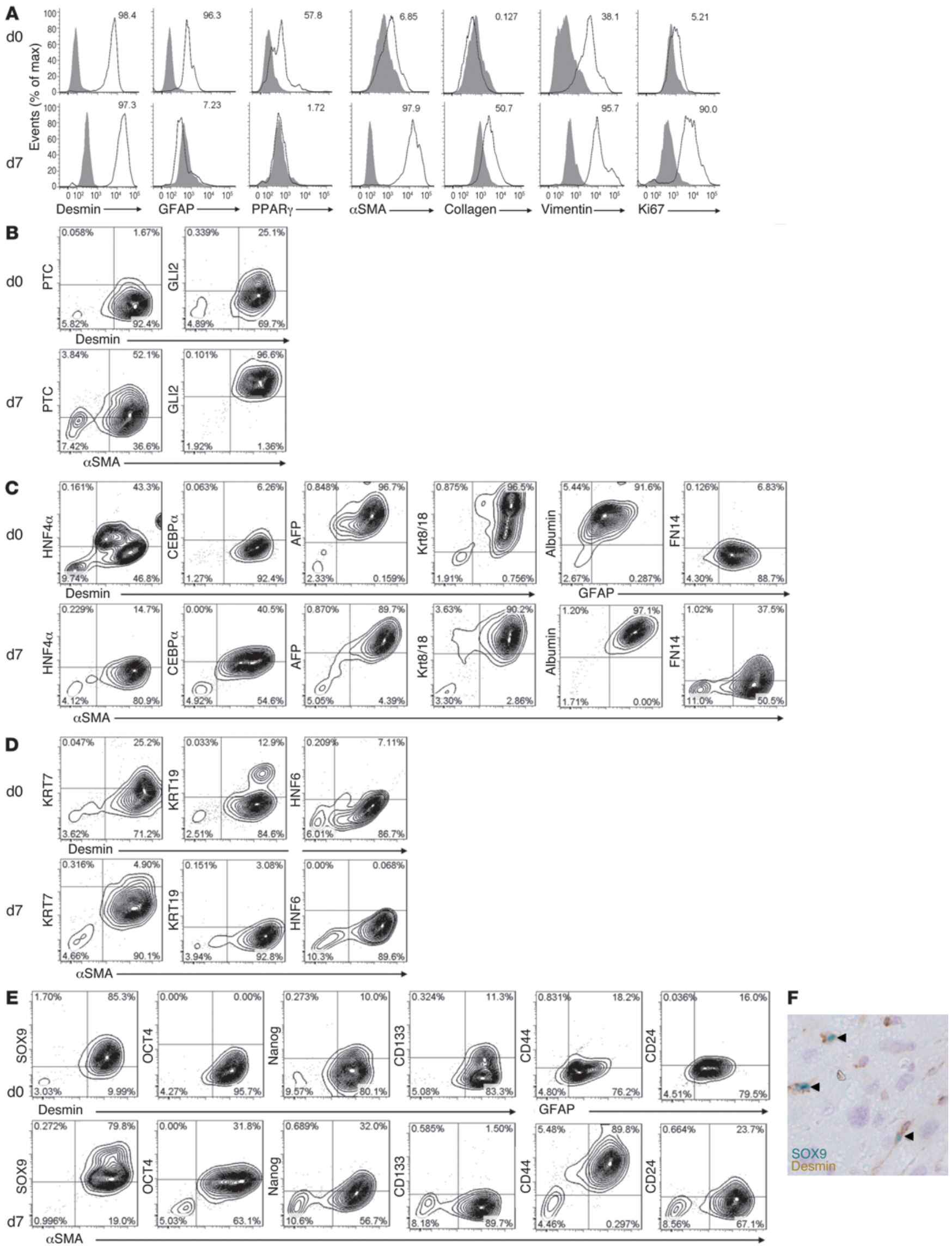


\section{Figure 7}

FACS evidence that HSCs and MF-HSCs are reprogrammable. (A) Intracellular staining of stellate cell markers desmin, GFAP, PPAR $\gamma$, $\alpha S M A$, collagen, vimentin, and Ki67 on freshly isolated murine HSCs (day 0 mouse HSCs) and MF-HSCs after 7 days of culture (day 7 mouse HSCs). Shaded areas indicate isotype controls. Costaining of desmin, GFAP, or $\alpha$ SMA on day 0 or day 7 mouse HSCs with (B) Hh signaling factors (PTC1, GLI2), (C) markers of hepatocytes and/or their progenitors (HNF4 $\alpha, \mathrm{CCAAT} /$ enhancer-binding protein $\alpha$ [CEBP $\alpha$ ], AFP, Krt8/18, albumin, fibroblast growth factor-inducible molecule 14 [FN14]), (D) ductular cell markers (Krt7, Krt19, HNF6), and (E) progenitor markers (SOX9, OCT4, Nanog, CD133, CD44, CD24). (A-E) Percentages of cells positive for respective markers relative to isotype control are indicated. (F) Double immunohistochemistry for desmin (brown) and SOX9 (green) in WT (C57BL/6) livers demonstrates colocalization of these markers (arrowheads) (original magnification, $\times 100$ ).

in over $80 \%$ of HSCs regardless of culture (Figure 7E). Colocalization of this multipotent progenitor marker and a well-established HSC marker (desmin) was confirmed by immunostaining of both freshly isolated HSCs (Figure 8B) and intact liver sections (Figure 7F). Based on expression of other stem/progenitor-associated markers, such as OCT4 and Nanog, MF-HSCs were more enriched with primitive progenitor-type cells than freshly isolated HSCs (Figure 7, C-E, and Supplemental Figure 17). In contrast, cells expressing liver epithelial markers, such as $\mathrm{HNF} 4 \alpha$ and $\mathrm{Krt} 19$, were relatively enriched in fresh HSC isolates (Figure 7, C and D, and Supplemental Figure 17).

To determine whether the epithelioid cells in the freshly isolated HSC preparations were quiescent HSCs, we evaluated the cells for coexpression of albumin and quiescent HSC markers (desmin or GFAP), reasoning that albumin is a relatively specific marker for hepatocytes and their immediate precursors $(45,46)$. Flow cytometry demonstrated coexpression of albumin and desmin in over $90 \%$ of the freshly isolated HSCs (Figure 7C). qRT-PCR analysis of freshly isolated HSCs also demonstrated albumin mRNA (Figure 8A), and in situ hybridization verified coexpression of albumin and GFAP transcripts (Figure 8B). This direct evidence for albumin expression in freshly isolated HSCs complements data that desmin ${ }^{+}$cells coexpress E-cadherin (Supplemental Figure 2) and, together with the other flow cytometry and PCR data (Figures 7 and 8 and Supplemental Figures 16 and 17), confirm that Q-HSCs are epithelioid cells with characteristics of hepatocyte progenitors.

It is well accepted that Q-HSCs become MFs in injured livers and during culture. Therefore, we examined day 7 culture-activated HSCs for evidence of albumin expression. We were unable to demonstrate albumin transcripts in day 7 HSCs using FISH (Supplemental Figure 18). Consistent with this, qRT-PCR confirmed that albumin mRNA levels were downregulated markedly in MF-HSCs relative to those in freshly isolated HSCs (Figure 8A). However, over $90 \%$ of the MF-HSCs coexpressed albumin and aSMA by flow cytometry (Figure 7C). While it is possible that cell-adherent albumin from the culture medium explains the discordant flow cytometry and mRNA data, it is also conceivable that MF-HSCs retain albumin that they generated at an earlier time point given the long half-life of albumin protein.

The latter possibility implies that MF-HSCs would exhibit other independent proof of previous albumin gene expression. Therefore, we examined HSCs that were harvested from IKK $\beta^{\Delta \text { Hep mice, }}$ a strain in which regulatory elements of the albumin gene drive expression of Cre-recombinase, disrupting the inhibitor $\kappa \beta$ kinase $\beta(I K K \beta)$ gene in hepatocytes (47). Mutant IKK $\beta$ and Cre recombinase were evident in both RNA and DNA from freshly isolated HSCs and culture-activated primary MF-HSCs from IKK $\beta^{\Delta \text { Hep }}$ mice, whereas neither the mutant allele, nor Cre, could be demonstrated in HSCs that were harvested from IKK $\beta^{\mathrm{fl} / \mathrm{fl}}$ control mice (Supplemental Figure 19). These findings were replicated using HSCs from mice that had been subjected to BDL to stimulate in vivo reprogramming of HSCs into MFs. While it is conceivable that freshly isolated HSC preparations might harbor contaminating hepatocytes, hepatocytes do not survive 7-day culture under conditions that are routinely used to induce HSC transdifferentiation into MFs. Thus, the results prove that murine MF-HSCs are derived from cells with sufficient endogenous albumin transcriptional activity to drive expression of Cre recombinase and affect $I K K \beta$ gene rearrangement.

To evaluate the generalizability of these data, we performed FACS analysis of 2 clonal myofibroblastic HSC lines. The majority of clonal human MF-HSCs (LX2 cells) and clonal rat MF-HSCs (8B cells) were Hh-responsive and coexpressed albumin and aSMA. Highly prevalent coexpression of AFP and $\alpha$ SMA, as well as coexpression $\alpha$ SMA and various other stem/progenitor markers, was likewise demonstrated (Supplemental Figures 20 and 21).

Although our FACS findings supported results generated by our fate-mapping studies in intact $\alpha$ SMA-Cre-ER ${ }^{\mathrm{T} 2} / \mathrm{ROSA}$-Stop-floxYFP DTG mice and GFAP-Cre-ERTM/ROSA-Stop-flox-YFP DTG mice (Figure 6, A and D), evidence that HSCs can become both MFs and liver epithelial cells seems to refute reports that mesoderm gives rise to HSCs during embryogenesis (48) as well as claims that epithelial-mesenchymal transitions do not occur in adult livers $(42,43,49)$. Therefore, it was critical to determine whether our findings could be confirmed by an alternative approach. To do this, we performed lineage tracing in a fourth DTG line (GFAPCre/ROSA-Stop-flox-LacZ mice), in which constitutive GFAP expression in healthy adult mice is used to mark GFAP-expressing cells and their progeny. HSCs and cells lining canals of Hering (an acknowledged reservoir of SOX9 $9^{+}$bipotent liver epithelial progenitors) are $\mathrm{LacZ}^{+}$in these mice (8). LacZ staining was also demonstrated throughout the extrahepatic biliary tree, gall bladder, and pancreatic ducts (Supplemental Figure 22). All of the latter structures are also known to derive from SOX9-positive multipotent progenitors (32). SOX9 interacts with the Hh pathway to control progenitor fate decisions $(50,51)$, suggesting that some level of basal Hh signaling might be evident in the healthy adult pancreatobiliary tree. To address this issue, we stained tissues from PTCLac $Z$ mice in which expression of $\beta$-galactosidase is controlled by promoter elements of the Hh target gene, Ptc (7). Faint LacZ staining was evident in the gall bladder, extrahepatic bile duct, and pancreatic ducts (Supplemental Figure 22).

\section{Discussion}

This study proves that SMO, an obligate intermediate in the canonical Hh signaling pathway, controls the injury-related accumulation of MFs and liver progenitors in adult liver. Moreover, the results uncover what we believe to be a novel mechanism for replacing injured hepatic epithelia that depends upon SMOdirected modulation of epithelial-mesenchymal transitions in HSCs, the resident liver pericyte that is a major source of MFs during many types of liver injury. 
A
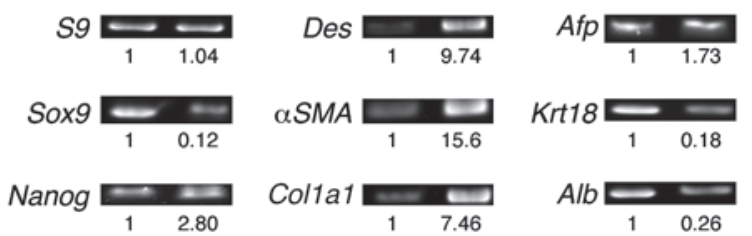

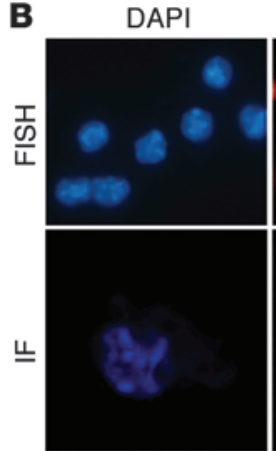

DAPI

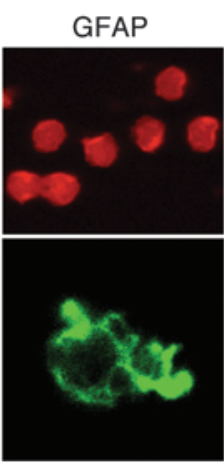

Desmin

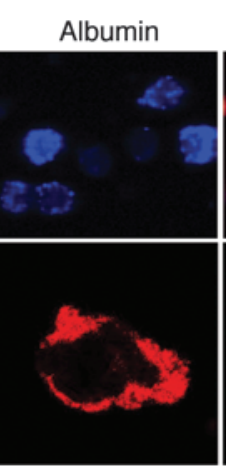

Albumin

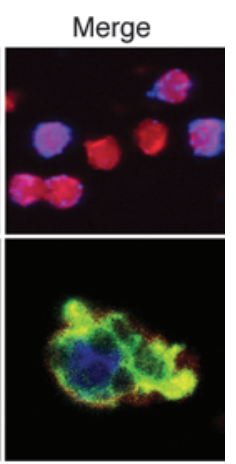

Merge
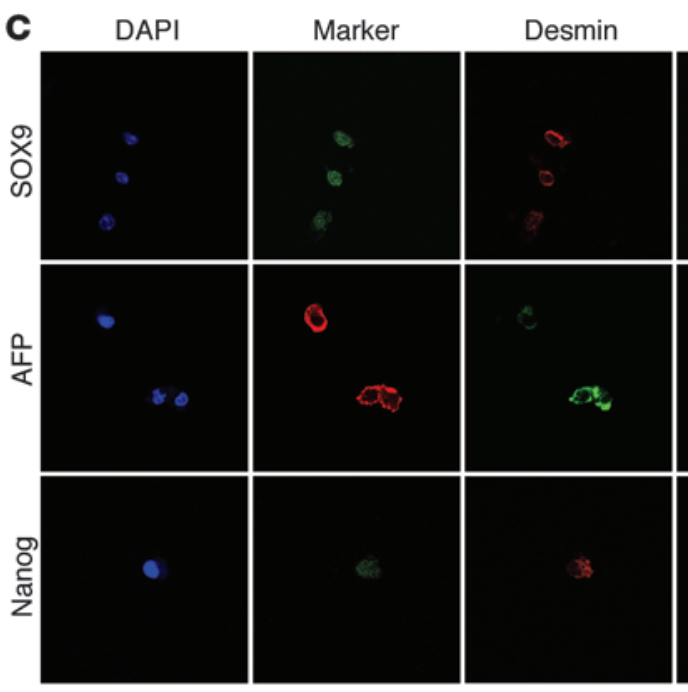

Day 0
Merge
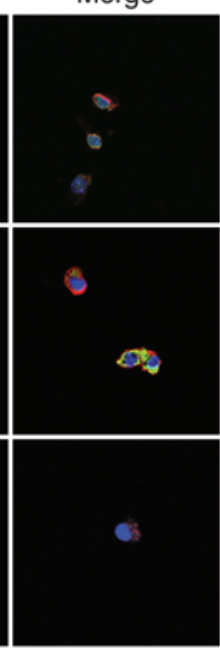

2
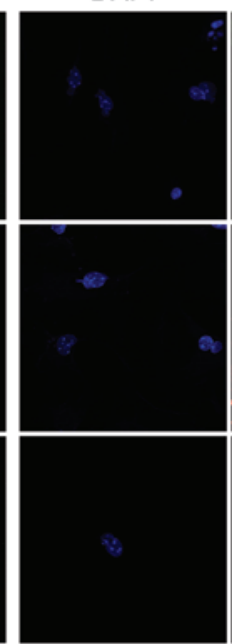

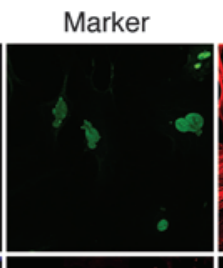

ASMA

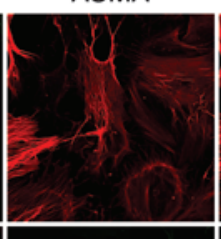

Merge
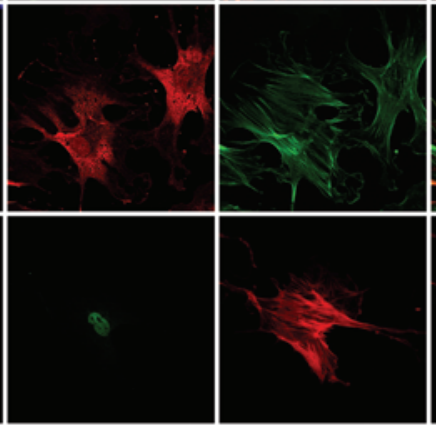

Day 7

Figure 8

HSCs differentially coexpress markers of hepatocytes, progenitors, and MFs. (A) HSCs were isolated from WT mice. Total RNA was purified from either freshly isolated cells or cells cultured for 7 days and analyzed by RT-PCR. Amplicons were visualized by agarose gel electrophoresis. Band intensity was quantified and expressed relative to day 0 mRNA level. (B) FISH for GFAP (red) and albumin (blue) mRNAs in freshly isolated Q-HSCs (original magnification, $\times 100$ ) (top). Immunofluorescence (IF) staining demonstrates colocalization of desmin (green) and albumin proteins in day 0 HSCs visualized by confocal microscopy. Nuclei are demonstrated by DAPI staining (original magnification, $\times 100)($ bottom). (C) Expression of representative hepatocytic and progenitor markers (AFP, SOX9, Nanog) in day 0 HSCs (desmin-positive) and day 7 cultureactivated mouse HSCs ( $\alpha$ SMA-positive) visualized by confocal microscopy. Nuclei are demonstrated by DAPI staining (original magnification, $\times 100$ [day 0 mouse HSCs]; $\times 40$ [day 7 mouse HSCs]).

These discoveries have several important implications. First, they show that a single molecule, SMO, ultimately coordinates fibrogenic and regenerative signals initiated when various injury-related factors interact with their receptors, because simply deleting SMO in MFs is sufficient to prevent hepatic accumulation of both MFs and liver epithelial progenitors. Albeit unexpected, this result is consistent with evidence that SMO integrates at least 3 major morphogenic signaling pathways, $\mathrm{Hh}$, Wnt, and TGF- $\beta(16,52)$, and indicates that SMO is a central regulator of both liver fibrosis and regeneration. This insight, in turn, identifies SMO as a therapeutic target to prevent cirrhosis and optimize recovery from liver damage. Second, proof of SMO's importance in adult liver repair validates the putative pathobiological significance of injury-related induction of Hh ligand production by liver epithelia and resultant hepatic accumulation of Hh-responsive MFs and progenitors (10). This justifies strategies that aim to prevent liver fibrosis by reducing hepatic epithelial injury, since success aborts production of Hh ligands that would otherwise provide a major paracrine stimulus for SMO activation in MFs and consequent MF accumulation and liver fibrosis. Third, evidence that hepatic accumulation of liver epithelial progenitors is blocked (and liver atrophy enhanced) by conditional deletion of SMO in MFs provides an explanation for previous observations that MF depletion impairs liver regeneration in adults $(53,54)$ and implies that progenitors play a more important role in adult liver regeneration than generally believed. Fourth, proof that SMO-dependent signals are required for resident HSCs to become proliferative MFs, coupled with evidence that MF-HSCs express markers of multipotent progenitor cells, supports complementary lineage-tracing evidence that HSCs function as facultative progenitors to replace damaged liver epithelial cells in injured livers. 
Together, the data suggest a novel, and admittedly controversial, model whereby SMO dictates the outcome of liver injury by modulating the differentiation of a subpopulation of resident liver cells that retain multipotency. Recently, a subpopulation of transitional cells that coexpressed epithelial and mesenchymal markers was demonstrated in adult mammary glands. The epithelioid breast cells were capable of upregulating their expression of multipotency factors to acquire a more mesenchymal, stem cell-like phenotype. Transitional breast cells were particularly sensitive to reprogramming by virally delivered oncogenes. Spontaneous reprogramming also occurred, although the signals driving that process were not elucidated $(55,56)$. Our results support the concept that HSCs are the liver equivalent of the transitional breast cells and identify SMO activation as a key event in the reprogramming process. In healthy livers, which lack Hh ligands and other signals that activate SMO, HSCs are nonfibrogenic and nonproliferative (i.e., quiescent) pericytes. Like the transitional breast cells and pericytes in other tissues, these quiescent HSCs express some mesenchymal genes (55, 57-59). HSCs also express many markers of hepatocyte and cholangiocyte precursors, suggesting that they are poised to undergo MET if the need to replace mature liver epithelial cells arises acutely. Conversely, during chronic liver injury, the HSC microenvironment becomes progressively and persistently enriched with $\mathrm{Hh}$ ligands and other profibrogenic factors that activate SMO $(7,20)$. SMO activation in HSCs initiates signaling that downregulates epithelial genes but upregulates expression of genes that drive HSCs to become proliferative, myofibroblastic, and fibrogenic. Expression of pluripotency factors, such as Nanog and OCT4 (60), also emerges in some MF-HSCs. Thus, by generating sustained paracrine signals that persistently activate SMO in HSCs, chronic hepatic epithelial injury favors accumulation of both MFs and multipotent progenitors, while suppressing the differentiation of these multipotent progenitors into quiescent HSCs and more mature liver epithelial cells. Although these responses might be viewed as an appropriate adaptation that assures eventual replacement of hepatic epithelia once hepatotoxic threats dissipate, fibrosis ensues as the liver becomes progressively repopulated by relatively undifferentiated and myofibroblastic multipotent progenitors and their immediate progeny.

This model does not preclude roles for other types of liver epithelial progenitor cells in maintaining hepatic epithelial homeostasis. Indeed, the latter is likely given strong evidence that hepatic progenitor populations are heterogeneous and diverse situations evoke a demand for replacement of hepatic epithelial cells (61). Nor does the model define the ultimate source of HSCs. It is fully consistent with the possibility that adult HSCs derive from putative precursors in either the adult biliary tree $(33,61,62)$ or vestigial mesothelium $(48,63)$. There is also room for MFs derived from cells other than HSCs in the new model. Unlike MF-HSCs, which are capable of transitioning back into nonfibrogenic cells, PFs and bone marrow-derived fibrocytes appear to provide a consistent source of collagen matrix (26). The relative significance of different MF populations is already believed to vary according to the type and duration of liver injury $(26,31)$. Further research is needed to clarify which types of MFs persist and continue to produce collagen matrix after epithelial injury subsides. Finally, the model has potential relevance for regeneration of other epithelial tissues with resident stellate cell/pericyte populations, such as adult pancreas and kidney (64). Our studies suggested that conditional deletion of SMO in $\alpha \mathrm{SMA}$-expressing cells reduced num- bers of renal $\alpha \mathrm{SMA}^{+}$cells (Supplemental Figure 10). If stellate cells/pericytes in those tissues also retain sufficient plasticity to transition into replacement epithelial cells, then SMO might be a master regulator of epithelial repair in adults. Moreover, because SMO activation is modulated by a number of different cell surface molecules that influence cell fate decisions (15), it may be a novel "druggable" target to prevent fibrosis and assure regeneration of healthy epithelia during many types of tissue damage.

\section{Methods}

Animals and experimental design. Smo ${ }^{\text {tm2Amc }} / \mathrm{J}$ (SMO-flox) mice (65) and ROSAStop-flox-YFP mice (38) were obtained from The Jackson Laboratory and were crossed with $\alpha$ SMA-Cre-ER ${ }^{\mathrm{T} 2}$ transgenic mice (24), which express TMX -regulated Cre recombinase under control of the $\alpha$ SMA promoter. DTG aSMA-Cre $\times$ SMO/flox homozygote control mice were bred by crossing $\mathrm{SMO} /$ flox homozygote, $\alpha \mathrm{SMA}-\mathrm{Cre}-\mathrm{ER}^{\mathrm{T} 2}$ hemizygous mice with SMO/flox homozygote mice. Additionally, $\alpha$ SMA-Cre-ER ${ }^{\mathrm{T} 2} \times$ ROSA-Stop-flox-YFP mice were bred by crossing ROSA-Stop-flox-YFP homozygote, $\alpha$ SMA-Cre$\mathrm{ER}^{\mathrm{T} 2}$ hemizygous mice with ROSA-Stop-flox-YFP homozygote mice. Adult (aged 8-12 weeks) mice were subjected to either BDL or sham surgery, as described previously (66) ( $n=11$ mice per group). Animals were killed 14 days after surgery; blood and liver samples were obtained.

To induce oxidative liver injury and activate HSC and liver progenitor populations, $\alpha \mathrm{SMA}-\mathrm{Cre}-\mathrm{ER}^{\mathrm{T} 2} \times \mathrm{SMO} /$ flox DTG mice were fed a methionine/choline-deficient diet supplemented with $0.15 \%$ ethionine (MCDE), as described previously (7). Animals were divided into groups that received either vehicle $(n=5)$ or TMX $(n=6)$, and surviving mice were sacrificed after being fed MCDE diets for 1 week. Chow-fed $\alpha$ SMA-Cre-ER ${ }^{\mathrm{T} 2} \times \mathrm{SMO} /$ flox DTG mice treated either with vehicle $(n=6)$ or TMX $(n=6)$ were also sacrificed at the same time point.

IKK $\beta^{\mathrm{fl} / \mathrm{fl}}$ and IKK $\beta^{\Delta \mathrm{Hep}}$ mice were gifts from Michael Karin (UCSD, San Diego, California, USA). IKK $\beta^{\mathrm{f} / / \mathrm{fl}}$ mice were bred with albumin-Cre mice to generate animals in which albumin-expressing cells and their progeny are IKK $\beta$ deficient (i.e., IKK $\beta^{\Delta \mathrm{Hep})}$ (67). All mice were housed in a facility with a 12-hour-light/dark cycle and allowed free access to food and water. Both single-transgenic IKK $\beta^{\mathrm{fl} / \mathrm{fl}}$ and DTG IKK $\beta^{\Delta \mathrm{Hep}}$ animals were subjected to $\mathrm{BDL}$, as described above ( $n=4$ mice per group).

ROSA-Stop-flox-LacZ mice were obtained from The Jackson Laboratory (http://www.jax.org). Mice expressing Cre recombinase under the control of the constitutive GFAP regulatory element (GFAP-Cre-ER mice) were crossed with ROSA-Stop-flox-LacZ transgenic mice, which have been previously described (8). Adult male PTC-LacZ reporter mice have been previously described (18).

TMX injection. To initiate Cre-mediated gene rearrangement of floxed alleles, DTG mice subjected to either sham operation or BDL (approximately $25 \mathrm{~g}$ in body weight) were injected intraperitoneally with TMX (Sigma-Aldrich) at $10 \mathrm{mg}$ per $\mathrm{kg}$ body weight. Injections for BDL injuries were initiated at day 0 for GFAP mice and day 4 for $\alpha$ SMA mice. Injections were delivered every other day until tissues were harvested at day 14 after surgery. For MCDE diet-induced injury, TMX was injected beginning at day 0 of MCDE diet administration and delivered every other day until tissue was harvested at day 7 .

$\beta$-Galactosidase staining. Staining of $\beta$-galactosidase in whole-mount tissue and in paraffin-embedded tissue was performed as previously described using the $\beta$-Galactosidase Detection Kit (Promega) (68).

Hydroxyproline assay. Hepatic hydroxyproline content was quantified colorimetrically in flash frozen liver samples, as described previously (7). Concentrations were calculated from a standard curve prepared with high-purity hydroxyproline (Sigma-Aldrich) and expressed as $\mathrm{mg}$ hydroxyproline per g liver. 
Immunohistochemistry. Liver tissue was fixed in formalin and embedded in paraffin. Immunohistochemical staining to detect GLI2, SMO, $\alpha$ SMA, elastin, Krt19, SOX9, AFP, desmin, Ki67, and cyclin D1 was performed using the DAKO Envision System (DAKO Corporation) according to the manufacturer's protocol. Immunostaining were performed as described previously (69). Briefly, formalin-fixed paraffin-embedded liver tissues were cut into $5-\mu \mathrm{m}$ sections and placed on glass slides. Sections were deparafinized with xylene, dehydrated with ethanol, and then incubated with $3 \%$ hydrogen peroxide to block endogenous peroxidase. Antigen retrieval was performed by heating in $10 \mathrm{mM}$ sodium citrate buffer ( $\mathrm{pH} 6.0$ ) or incubating with pepsin (00-3009; Invitrogen). Sections were blocked in DAKO protein block (X9090; DAKO), followed by incubation with primary antibodies. The following primary antibodies were used: GLI2 (GWB-EB3B44; 1:4,500; GenWay Biotech), desmin (ab6322; 1:1,000; Abcam), elastin (BA-4; 1:2,000; Santa Cruz Biotechnology Inc.), Krt19 (Troma III, 1:400; Hydroma Bank), AFP (A0008, 1:400; DAKO), aSMA (M0851; 1:500; DAKO), SOX9 (AB5535; 1:1,000; Millipore), Ki67 (NCLL-Ki67-MM1; 1:1,000; Novocast), cyclin D1 (ab16663; 1:1,000; Abcam); SMO (sc-6366; 1:150; Santa Cruz Biotechnology Inc.); YFP (GFP-1020; 1:600; Aves Labs); and GFAP (DAKO; 1:1,000; Novus). HRP-conjugated anti-rabbit (K4003; DAKO), anti-mouse (K4001; DAKO) secondary antibodies were used to visualize target proteins. DAB reagent (K3466; DAKO) was applied in the detection procedure. Tissue sections were counterstained with Aqua Hematoxylin-INNOVEX (Innovex Biosciences). Double immunohistochemistry was performed using Vina Green, according to the manufacturer's recommendation (BioCare Medical). Negative controls included liver specimens exposed to $1 \%$ bovine serum albumin instead of the respective primary antibodies.

Sirius red staining and $\alpha \mathrm{SMA}$, desmin, and elastin immunohistochemical staining were assessed by morphometry (MetaView software, Universal Imaging Corp.). For morphometric quantification, 10 randomly chosen fields at $\times 20$ magnification per section were evaluated for each mouse. GLI2, SOX9, Ki67, and cyclin D1 staining was quantified using similar approaches: for each of these parameters, numbers of cells with stained nuclei were counted in 10 randomly chosen $\times 20$ fields per section per mouse.

In situ hybridization. To detect specific transcripts within isolated HSCs and hepatocytes, FISH was carried out using validated probes specific for mouse albumin (VB6-12839, Panomics), collagen 1 1 1 (VB1-13595, Panomics), and GFAP (VB1-10623, Panomics) mRNAs, according to the manufacturer's protocol. Briefly, freshly isolated HSCs and hepatocytes, or day 7 culture activated HSCs, were fixed on cover slips using $4 \%$ formaldehyde. Cells were permeabilized and hybridized with probes indicated in Figure 8B. Q-HSC multiplex hybridizations were GFAP (Cy3) and albumin (Cy5). MF-HSC multiplex hybridizations were COL1A1 (Cy3) and albumin (Cy5), and hepatocytes were probed with COL1A1 (Cy3) and albumin (Cy5). Slides were then mounted in Vectashield Aqueous Mounting Media with DAPI (Vector Laboratories). Slides were viewed with a Zeiss 710 inverted confocal microscope through the Duke Confocal Microscopy Core Facility.

Immunocytochemistry. HSCs were cytospun or grown on coverslips for 7 days. Cells were washed, fixed in $4 \%$ paraformaldehyde and permeabilized with cold methanol, and incubated with primary antibodies for 2 hours at room temperature. Cells were washed in PBS and incubated with Alexa Fluor 594 goat anti-mouse IgG or Alexa Fluor 488 goat anti-rabbit $\operatorname{IgG}(\mathrm{H}+\mathrm{L})$ for 45 minutes at room temperature. Slides were mounted on slides with Prolong Gold Antifade Reagent (Invitrogen) and viewed with a Zeiss 710 inverted confocal microscope (Carl Zeiss).

HSC isolation and culture. Primary HSCs were isolated from DTG mice as well as IKK $\beta^{\mathrm{fl} / \mathrm{fl}}$ and IKK $\beta^{\Delta \mathrm{Hep}}$ mice. Isolated HSCs were assessed for purity and viability (Supplemental Figure 16C) and seeded at a density of $3 \times 10^{2}$ cells per $\mathrm{mm}^{2}$ in DMEM supplemented with $10 \%$ fetal bovine serum and penicillin/streptomycin, as described previously (11).

Adenoviral transduction of HSCs. Adenoviruses, harboring either the GFP gene (AdGFP) or Cre recombinase gene (AdCre), were added to primary murine HSCs at culture day 4 at a MOI of 50, as described previously (11). After 24 hours, virus-containing medium was aspirated and replaced with fresh medium. Viral efficiency of AdGFP infection was assessed by confocal microscopy, with $>95 \%$ of infected cells found to be GFP positive. Cre expression of infected cells was confirmed by Western analysis of cellular whole cell extracts.

Measures of injury. Serum alanine aminotransferase and triglycerides were measured using commercially available kits from Biotron Diagnostics (66-D) and Cayman Chemicals (10010303), respectively, according to the manufacturer's instructions. TUNEL assays were performed on paraffin-embedded tissue using a commercially available assay from Roche Applied Science (In Situ Cell Death Detection Assay, 11-684-817-910)

Cell line maintenance. Human MF-HSC line LX2 (provided by Scott Friedman, Mount Sinai School of Medicine, New York, New York, USA) (70) was cultured in Dulbecco's modified Eagle's medium, supplemented with $10 \%$ fetal calf serum, $1 \mathrm{mM}$ L-glutamine, and $100 \mathrm{IU} / \mathrm{ml}$ penicillin/streptomycin.

Clonally derived rat MF-HSC line 8B (71) was cultured in RPMI 1640 medium and supplemented with $10 \%$ fetal calf serum and $100 \mathrm{IU} / \mathrm{ml}$ penicillin/streptomycin. All cells were harvested by trypsinization and used for flow cytometry analysis. Phenotypic characterization of all HSC lines is provided in Supplemental Table 1

Two-step real-time RT-PCR and conventional RT-PCR. Total RNA was extracted from cells, livers, or brains using TRIzol (Invitrogen), followed by RNase-free DNase I treatment (Qiagen). RNA was reverse transcribed to cDNA templates using random primer and SuperScript RNase H-Reverse Transcriptase (Invitrogen) and amplified.

For semiquantitative qRT-PCR, $1.5 \%$ of the first-strand reaction was amplified using StepOne Plus Real-Time PCR Platform (ABI/Life Technologies) and specific oligonucleotide primers for target sequences as well as the ribosomal S9 housekeeping gene. qRT-PCR parameters were as follows: denaturating at $95^{\circ} \mathrm{C}$ for 3 minutes, followed by 40 cycles of denaturing at $95^{\circ} \mathrm{C}$ for 10 seconds and annealing/extension at the optimal primers temperatures for 60 seconds. Threshold cycles $\left(C_{t}\right)$ were automatically calculated by the StepOne Plus Real-Time Detection System. Target gene levels in the cells are presented as a ratio to levels detected in the corresponding control cells according to the $2^{-\Delta \Delta C t}$ method. Primer sequences are listed in Supplemental Table 3.

Flow cytometry. All the staining steps were performed in the dark at $4^{\circ} \mathrm{C}$ and blocked with BD Fc Block. For surface staining, cells were incubated for 30 minutes with antibody in flow cytometry staining buffer (eBioscience) and were washed 3 times with flow cytometry buffer. For intracellular staining, cells were fixed and permeabilized for 20 minutes with Cytofix/ Cytoperm buffer (BD PharMingen) and were washed twice with Perm/ Wash buffer (BD PharMingen), followed by incubation with antibody in Perm/Wash buffer for 30 minutes. LSR II (BD) and FACSDiva software (BD) were used for the acquisition of flow cytometry data, and FlowJo software (TreeStar) was used for analysis. BODIPY staining for lipid content was performed by incubating mouse HSCs with BODIPY 493/503 (4,4-difluoro-1,3,5,7,8-pentamethyl-4-bora-3a,4a-diaza-s-indacene) $(20 \mu \mathrm{g} / \mathrm{ml}$; Invitrogen) for 30 minutes and was analyzed using FITC channel. YFP expression in hepatocytes was analyzed using FITC channel. Antibodies and conjugates are listed in Supplemental Table 2.

Statistics. All data were expressed as mean \pm SEM. Statistical analysis was performed using Student's $t$ test. All analysis was conducted using GraphPad Prism 4 software (GraphPad Software Inc.). Differences with $P \leq 0.05$ were considered to be statistically significant. 
Study approval. Animal care and surgical procedures were approved by the Duke University Medical Center Institutional Animal Care and Use Committee as set forth in the Guide for the Care and Use of Laboratory Animals published by the NIH.

\section{Acknowledgments}

This work was supported in part by NIH grants R37 AA010154 and R01 DK077794 (to A.M. Diehl).

1. Pinzani M, Rosselli M, Zuckermann M. Liver cirrhosis. Best Pract Res Clin Gastroenterol. 2011;25(2):281-290.

2. Lee UE, Friedman SL. Mechanisms of hepatic fibrogenesis. Best Pract Res Clin Gastroenterol. 2011;25(2):195-206.

3. Wells RG. Cellular sources of extracellular matrix in hepatic fibrosis. Clin Liver Dis. 2008;12(4):759-768, viii.

4. Hernandez-Gea V, Friedman SL. Pathogenesis of liver fibrosis. Annu Rev Pathol. 2011;6:425-456.

5. Roskams T. Relationships among stellate cell activation, progenitor cells, and hepatic regeneration. Clin Liver Dis. 2008;12(4):853-860, ix.

6. Hinz B, et al. Recent developments in myofibroblast biology: paradigms for connective tissue remodeling. Am J Pathol. 2012;180(4):1340-1355.

7. Jung Y, et al. Signals from dying hepatocytes trigger growth of liver progenitors. Gut. 2010;59(5):655-665.

8. Yang L, et al. Fate-mapping evidence that hepatic stellate cells are epithelial progenitors in adult mouse livers. Stem Cells. 2008;26(8):2104-2113.

9. Beachy PA, Hymowitz SG, Lazarus RA, Leahy DJ, Siebold C. Interactions between Hedgehog proteins and their binding partners come into view. Genes Dev. 2010;24(18):2001-2012.

10. Omenetti A, Choi S, Michelotti G, Diehl AM. Hedgehog signaling in the liver. J Hepatol. 2011; 54(2):366-373.

11. Choi SS, et al. Leptin promotes the myofibroblastic phenotype in hepatic stellate cells by activating the hedgehog pathway. $J$ Biol Chem. 2010;285(47):36551-36560.

12. Omenetti A, et al. Hedgehog-mediated mesenchymal-epithelial interactions modulate hepatic response to bile duct ligation. Lab Invest. 2007;87(5):499-514.

13. Yang $\mathrm{L}$, et al. Sonic hedgehog is an autocrine viability factor for myofibroblastic hepatic stellate cells. J Hepatol. 2008;48(1):98-106.

14. Omenetti A, Diehl AM. Hedgehog signaling in cholangiocytes. Curr Opin Gastroenterol. 2011; 27(3):268-275

15. Chen W, et al. Activity-dependent internalization of smoothened mediated by beta-arrestin 2 and GRK2. Science. 2004;306(5705):2257-2260.

16. Javelaud D, Alexaki VI, Dennler S, Mohammad KS, Guise TA, Mauviel A. TGF-beta/SMAD/GLI2 signaling axis in cancer progression and metastasis. Cancer Res. 2011;71(17):5606-5610.

17. Fan $\mathrm{Q}$, et al. Requirement of TGFbeta signaling for SMO-mediated carcinogenesis. J Biol Chem. 2010;285(47):36570-36576.

18. Sicklick JK, et al. Dysregulation of the Hedgehog pathway in human hepatocarcinogenesis. Carcinogenesis. 2006;27(4):748-757.

19. Ochoa B, et al. Hedgehog signaling is critical for normal liver regeneration after partial hepatectomy in mice. Hepatology. 2010;51(5):1712-1723.

20. Philips GM, et al. Hedgehog signaling antagonist promotes regression of both liver fibrosis and hepatocellular carcinoma in a murine model of primary liver cancer. PLoS One. 2011;6(9):e23943.

21. Higashi N, Kojima N, Miura M, Imai K, Sato M, Senoo H. Cell-cell junctions between mammalian (human and rat) hepatic stellate cells. Cell Tissue Res. 2004;317(1):35-43.

22. Lim YS, Lee HC, Lee HS. Switch of cadherin
Received for publication September 17, 2012, and accepted in revised form February 12, 2013.

Address correspondence to: Anna Mae Diehl, Division of Gastroenterology, Duke University Medical Center, 595 LaSalle Street, Snyderman Building, Suite 1073, Durham, North Carolina 27710, USA. Phone: 919.684.4173; Fax: 919.684.4183; E-mail: diehl004@ mc.duke.edu. expression from E- to $\mathrm{N}$-type during the activation of rat hepatic stellate cells. Histochem Cell Biol. 2007;127(2):149-160.

23. Cho IJ, et al. E-cadherin antagonizes transforming growth factor beta1 gene induction in hepatic stellate cells by inhibiting RhoA-dependent Smad3 phosphorylation. Hepatology. 2010;52(6):2053-2064.

24. Wendling O, Bornert JM, Chambon P, Metzger D. Efficient temporally-controlled targeted mutagenesis in smooth muscle cells of the adult mouse. Genesis. 2009;47(1):14-18.

25. Li Z, Dranoff JA, Chan EP, Uemura M, Sevigny J, Wells RG. Transforming growth factor-beta and substrate stiffness regulate portal fibroblast activation in culture. Hepatology. 2007;46(4):1246-1256.

26. Iwaisako K, Brenner DA, Kisseleva T. What's new in liver fibrosis? The origin of myofibroblasts in liver fibrosis. J Gastroenterol Hepatol. 2012;2:65-68.

27. Zhang L, Theise N, Chua M, Reid LM. The stem cell niche of human livers: symmetry between development and regeneration. Hepatology. 2008; 48(5):1598-1607.

28. Li MK, Crawford JM. The pathology of cholestasis. Semin Liver Dis. 2004;24(1):21-42.

29. Durnez A, et al. The clinicopathological and prognostic relevance of cytokeratin 7 and 19 expression in hepatocellular carcinoma. A possible progenitor cell origin. Histopathology. 2006;49(2):138-151.

30. Novo E, di Bonzo LV, Cannito S, Colombatto S, Parola M. Hepatic myofibroblasts: a heterogeneous population of multifunctional cells in liver fibrogenesis. Int J Biochem Cell Biol. 2009;41(11):2089-2093.

31. Dranoff JA, Wells RG. Portal fibroblasts: Underappreciated mediators of biliary fibrosis. Hepatology. 2010;51(4):1438-1444.

32. Furuyama K, et al. Continuous cell supply from a Sox9-expressing progenitor zone in adult liver, exocrine pancreas and intestine. Nat Genet. 2011;43(1):34-41.

33. Cardinale V, et al. Multipotent stem/progenitor cells in human biliary tree give rise to hepatocytes, cholangiocytes and pancreatic islets. Hepatology. 2011;54(6):2159-2172.

34. Dorrell C, et al. Prospective isolation of a bipotential clonogenic liver progenitor cell in adult mice. Genes Dev. 2011;25(11):1193-1203.

35. Carpino G, et al. Biliary tree stem/progenitor cells in glands of extrahepatic and intraheptic bile ducts: an anatomical in situ study yielding evidence of maturational lineages. J Anat. 2012;220(2):186-199.

36. Kalinichenko VV, et al. Ubiquitous expression of the forkhead box M1B transgene accelerates proliferation of distinct pulmonary cell types following lung injury. J Biol Chem. 2003;278(39):37888-37894.

37. Nelsen CJ, Rickheim DG, Tucker MM, Hansen LK, Albrecht JH. Evidence that cyclin D1 mediates both growth and proliferation downstream of TOR in hepatocytes. J Biol Chem. 2003;278(6):3656-3663.

38. Srinivas S, et al. Cre reporter strains produced by targeted insertion of EYFP and ECFP into the ROSA26 locus. BMC Dev Biol. 2001;1:4.

39. Hinz B, Phan SH, Thannickal VJ, Galli A, Bochaton-Piallat ML, Gabbiani G. The myofibroblast: one function, multiple origins. Am J Pathol. 2007;170(6):1807-1816

40. Kordes C, et al. CD133+ hepatic stellate cells are progenitor cells. Biochem Biophys Res Commun.
2007;352(2):410-417.

41. Hanley KP, Oakley F, Sugden S, Wilson DI, Mann DA, Hanley NA. Ectopic SOX9 mediates extracellular matrix deposition characteristic of organ fibrosis. J Biol Chem. 2008;283(20):14063-14071.

42. Chu AS, et al. Lineage tracing demonstrates no evidence of cholangiocyte epithelial-to-mesenchymal transition in murine models of hepatic fibrosis. Hepatology. 2011;53(5):1685-1695.

43. Troeger JS, et al. Deactivation of hepatic stellate cells during liver fibrosis resolution in mice. Gastroenterology. 2012;143(4):1073-1083.

44. Kisseleva T, et al. Myofibroblasts revert to an inactive phenotype during regression of liver fibrosis. Proc Natl Acad Sci U S A. 2012;109(24):9448-9453.

45. Elmaouhoub A, Dudas J, Ramadori G. Kinetics of albumin- and alpha-fetoprotein-production during rat liver development. Histochem Cell Biol. 2007; 128(5):431-443.

46. Gailhouste L. Isolation and purification method of mouse fetal hepatoblasts. Methods Mol Biol. 2012;826:33-47.

47. Arkan MC, et al. IKK-beta links inflammation to obesity-induced insulin resistance. Nat Med. 2005;11(2):191-198.

48. Asahina K, Zhou B, Pu WT, Tsukamoto H. Septum transversum-derived mesothelium gives rise to hepatic stellate cells and perivascular mesenchymal cells in developing mouse liver. Hepatology. 2011;53(3):983-995.

49. Scholten D, et al. Genetic labeling does not detect epithelial-to-mesenchymal transition of cholangiocytes in liver fibrosis in mice. Gastroenterology. 2010;139(3):987-998.

50. Bien-Willner GA, Stankiewicz P, Lupski JR. SOX$9 \mathrm{cre} 1$, a cis-acting regulatory element located 1.1 $\mathrm{Mb}$ upstream of SOX9, mediates its enhancement through the SHH pathway. Hum Mol Genet. 2007;16(10):1143-1156.

51. Cervantes S, Lau J, Cano DA, Borromeo-Austin C, Hebrok M. Primary cilia regulate Gli/Hedgehog activation in pancreas. Proc Natl Acad Sci U S A. 2010;107(22):10109-10114.

52. Katoh Y, Katoh M. Hedgehog signaling, epithelial-to-mesenchymal transition and miRNA (review). Int J Mol Med. 2008;22(3):271-275.

53. Shen K, et al. Depletion of activated hepatic stellate cell correlates with severe liver damage and abnormal liver regeneration in acetaminophen-induced liver injury. Acta Biochim Biophys Sin (Shanghai). 2011; 43(4):307-315

54. Kalinichenko VV, et al. Foxf1 $1^{+/-}$mice exhibit defective stellate cell activation and abnormal liver regeneration following $\mathrm{CCl} 4$ injury. Hepatology. 2003;37(1):107-117.

55. Chaffer CL, et al. Normal and neoplastic nonstem cells can spontaneously convert to a stem-like state. Proc Natl Acad Sci U S A. 2011;108(19):7950-7955.

56. Scheel C, et al. Paracrine and autocrine signals induce and maintain mesenchymal and stem cell states in the breast. Cell. 2011;145(6):926-940.

57. Duffield JS. The elusive source of myofibroblasts: problem solved? Nat Med. 2012;18(8):1178-1180.

58. Duffield JS, Lupher M, Thannickal VJ, Wynn TA. Host responses in tissue repair and fibrosis. Annu Rev Pathol. 2013;8:241-276.

59. Hutchison N, Fligny C, Duffield JS. Resident 
mesenchymal cells and fibrosis. Biochim Biophys Acta. 2012;pii:S0925-4439(12)00275-X.

60. Heng JC, Orlov YL, Ng HH. Transcription factors for the modulation of pluripotency and reprogramming. Cold Spring Harb Symp Quant Biol. 2010;75:237-244.

61. Duncan AW, Dorrell C, Grompe M. Stem cells and liver regeneration. Gastroenterology. 2009;137(2):466-481.

62. Michalopoulos GK. Liver regeneration: alternative epithelial pathways. Int J Biochem Cell Biol. 2011;43(2):173-179.

63. Yin C, Evason KJ, Maher JJ, Stainier DY. The bHLH transcription factor Hand 2 marks hepatic stellate cells in zebrafish: Analysis of stellate cell entry into the developing liver. Hepatology. 2012;
56(5):1958-1970.

64. Nagy NE, et al. Storage of vitamin A in extrahepatic stellate cells in normal rats. J Lipid Res. 1997;38(4):645-658.

65. Long F, Zhang XM, Karp S, Yang Y, McMahon AP. Genetic manipulation of hedgehog signaling in the endochondral skeleton reveals a direct role in the regulation of chondrocyte proliferation. Development. 2001;128(24):5099-5108.

66 . Omenetti A, et al. Hedgehog signaling regulates epithelial-mesenchymal transition during biliary fibrosis in rodents and humans. J Clin Invest. 2008;118(10):3331-3342.

67. Beraza N, Ludde T, Assmus U, Roskams T, Vander Borght S, Trautwein C. Hepatocyte-specific IKK gamma/NEMO expression determines the degree of liver injury. Gastroenterology. 2007;132(7):2504-2517.

68 . Sicklick JK, et al. Hedgehog signaling maintains resident hepatic progenitors throughout life. Am J Physiol Gastrointest Liver Physiol. 2006;290(5):G859-G870.

69. Omenetti A, et al. Paracrine modulation of cholangiocyte serotonin synthesis orchestrates biliary remodeling in adults. Am J Physiol Gastrointest Liver Physiol. 2011;300(2):G303-G315.

70. Xu L, et al. Human hepatic stellate cell lines, LX-1 and LX-2: new tools for analysis of hepatic fibrosis. Gut. 2005;54(1):142-151.

71. Greenwel P, Schwartz M, Rosas M, Peyrol S, Grimaud JA, Rojkind M. Characterization of fat-storing cell lines derived from normal and CCl4-cirrhotic livers. Differences in the production of interleukin-6. Lab Invest. 1991;65(6):644-653. 